\title{
Cancer-associated stroke: Pathophysiology, detection and management (Review)
}

\author{
EFTHIMIOS DARDIOTIS $^{1}$, ATHINA-MARIA ALOIZOU ${ }^{1 *}$, SOFIA MARKOULA $^{2 *}$, VASILEIOS SIOKAS $^{1}$, \\ KONSTANTINOS TSAROUHAS ${ }^{3}$, GEORGIOS TZANAKAKIS ${ }^{4}$, MASSIMO LIBRA ${ }^{5}$, ATHANASSIOS P. KYRITSIS ${ }^{2}$, \\ ALEXANDROS G. BROTIS ${ }^{6}$, MICHAEL ASCHNER ${ }^{7}$, ILLANA GOZES ${ }^{8}$, DIMITRIOS P. BOGDANOS ${ }^{9,10}$, \\ DEMETRIOS A. SPANDIDOS ${ }^{11}$, PANAYIOTIS D. MITSIAS ${ }^{12,13}$ and ARISTIDIS TSATSAKIS ${ }^{14}$ \\ ${ }^{1}$ Department of Neurology, Laboratory of Neurogenetics, University of Thessaly, University Hospital of Larissa, \\ 41100 Larissa; ${ }^{2}$ Department of Neurology, University Hospital of Ioannina, 45110 Ioannina; ${ }^{3}$ Department of Cardiology, \\ University Hospital of Larissa, 41100 Larissa; ${ }^{4}$ Laboratory of Anatomy-Histology-Embryology, Medical School, \\ University of Crete, 71003 Heraklion, Greece; ${ }^{5}$ Department of Biomedical and Biotechnological Sciences, \\ Pathology and Oncology Section, University of Catania, 95124 Catania, Italy; ${ }^{6}$ Department of Neurosurgery, \\ University of Thessaly, University Hospital of Larissa, 41100 Larissa, Greece; ${ }^{7}$ Department of Molecular Pharmacology, \\ Albert Einstein College of Medicine, Bronx, NY 10461, USA; ${ }^{8}$ The Lily and Avraham Gildor Chair \\ for the Investigation of Growth Factors, The Elton Laboratory for Molecular Neuroendocrinology, \\ Department of Human Molecular Genetics and Biochemistry, Sackler Faculty of Medicine, Sagol School of \\ Neuroscience and Adams Super Center for Brain Studies, Tel Aviv University, Tel Aviv 69978, Israel; \\ ${ }^{9}$ Department of Rheumatology and Clinical Immunology, University General Hospital of Larissa, Faculty of Medicine, \\ School of Health Sciences, University of Thessaly, 40500 Larissa; ${ }^{10}$ Cellular Immunotherapy and \\ Molecular Immunodiagnostics, Biomedical Section, Centre for Research and Technology-Hellas (CERTH) - \\ Institute for Research and Technology-Thessaly (IRETETH), 41222 Larissa; ${ }^{11}$ Laboratory of Clinical Virology, and \\ ${ }^{12}$ Department of Neurology, School of Medicine, University of Crete, 71003 Heraklion, Greece; \\ ${ }^{13}$ Comprehensive Stroke Center and Department of Neurology, Henry Ford Hospital, Detroit, MI 48202, USA; \\ ${ }^{14}$ Laboratory of Toxicology, School of Medicine, University of Crete, 71003 Heraklion, Greece
}

Received November 23, 2018; Accepted December 28, 2018

DOI: 10.3892/ijo.2019.4669

Correspondence to: Dr Efthimios Dardiotis, Department of Neurology, Laboratory of Neurogenetics, University of Thessaly, University Hospital of Larissa, Biopolis, Mezourlo Hill, 41100 Larissa, Greece

E-mail: edar@med.uth.gr

*Contributed equally

Abbreviations: ADT, androgen deprivation therapy; ALL, acute lymphoblastic leukemia; AML, acute myeloid leukaemia; AUC, area under the curve; BSI, bloodstream infection; CLL, chronic lymphoblastic leukemia; CMV, cytomegalovirus; CNS, central nervous system; CRP, C-reactive protein; CSF, cerebrospinal fluid; CSFs, colony-stimulating factors; CT, computer-assisted tomography; CVD, cerebrovascular disease; CVE, cerebrovascular event; CVT, cerebrovascular thrombosis; DIC, disseminated intravascular coagulation; EDH, epidural hemorrhage; ESAs, erythropoiesis-stimulating agents; 18 -FDG, fludeoxyglucose $\left({ }^{18} \mathrm{~F}\right)$; GVHD, graft-versus-host disease; HGFs, hematopoietic growth factors; HSCT, hematopoietic stem cell transplantation; HSV, herpes simplex virus; HUS, hemolytic uremic syndrome; ICH, intracranial hemorrhage; ICP, intracranial pressure; IPH, intraparenchymal hemorrhage; ITH, intratumoral hemorrhage; IVL, intravascular lymphomatosis; LDH, lactate dehydrogenase; LMWHs, low-molecular-weight heparins; MDS, myelodysplastic syndrome;
Abstract. Numerous types of cancer have been shown to be associated with either ischemic or hemorrhagic stroke. In this review, the epidemiology and pathophysiology of stroke in cancer patients is discussed, while providing vital information on the diagnosis and management of patients with cancer and stroke. Cancer may mediate stroke pathophysiology either directly or via coagulation disorders that establish a state of hypercoagulation, as well as via infections. Cancer treatment

MRI, magnetic resonance imaging; MTX, methotrexate; NBTE, non-bacterial thrombotic endocarditis; NC, neoadjuvant chemotherapy; NCI, National Cancer Institute; NK, natural killer; NOAGs, new oral anticoagulants; PET, positron emission tomography; PPV, positive predictive value; RR, relative risk; RT, radiation therapy; rTPA, recombinant tissue plasminogen activator; SAH, subarachnoid hemorrhage; SDH, subdural hemorrhage; SVT, sinovenous thrombosis; TB, tuberculosis; TIA, transient ischemic attack; TTP, thrombotic thrombopenic purpura; VTE, venous thromboembolism

Key words: stroke, cancer, cancer-associated stroke, ischemic stroke, hemorrhagic stroke 
options, such as chemotherapy, radiotherapy and surgery have all been shown to aggravate the risk of stroke as well. The clinical manifestation varies greatly depending upon the underlying cause; however, in general, cancer-associated strokes tend to appear as multifocal in neuroimaging. Furthermore, several serum markers have been identified, such as high D-Dimer levels and fibrin degradation products. Managing cancer patients with stroke is a delicate matter. The cancer should not be considered a contraindication in applying thrombolysis and recombinant tissue plasminogen activator (rTPA) administration, since the risk of hemorrhage in cancer patients has not been reported to be higher than that in the general population. Anticoagulation, on the contrary, should be carefully examined. Clinicians should weigh the benefits and risks of anticoagulation treatment for each patient individually; the new oral anticoagulants appear promising; however, low-molecular-weight heparin remains the first choice. On the whole, stroke is a serious and not a rare complication of malignancy. Clinicians should be adequately trained to handle these patients efficiently.

\section{Contents}

1. Introduction

2. Cancer types associated with stroke

3. Pathophysiology

4. From stroke to cancer

5. Clinical presentation and diagnosis

6. Detection of a cancer-associated stroke

7. Management

8. Prevention

9. Conclusions

\section{Introduction}

Cerebrovascular diseases (CVDs) are common in cancer patients, significantly aggravating their condition and prognosis (1). Approximately $15 \%$ of cancer patients have a concomitant CVD $(2,3)$, and the frequency of cerebral infarcts is similar to that of cerebral hemorrhage (3). Stroke may either follow the initial cancer diagnosis (4) or may precede the diagnosis of cancerous disease (5). The prevalence of an underlying cancerous disorder is higher in patients with ischemic stroke than in the general population $(6,7)$ and cancer as a co-morbidity is found in 1 out of 10 hospitalized patients with ischemic stroke in the United States (8). Patients with cancer have been shown to have higher in-hospital post-stroke mortality rate $(1,9,10)$ and patients with ischemic stroke with an active cancer have also been found to be of younger age, with more severe and more frequent cryptogenic strokes (1).

The connection between stroke and cancer has for long captivated the interest of the medical community. The first large autopsy study was conducted in 1985 by Graus et al, showing that the most frequent complication of the central nervous system (CNS) in cancer patients, following metastasis, was cerebral infarction and hemorrhage. In the same study, $14.6 \%$ of cancer patients had pathological evidence of CVD and approximately half of them were symptomatic (11). More recent studies, such as that among Hodgkin lymphoma 5-year survivors, demonstrated that $7 \%$ of patients developed an ischemic stroke in the 17.5-year follow-up period (12).

The underlying causes for the development of a stroke in cancer patients differ from those of non-cancer patients, and are associated with the cancer itself, as well as with the type of treatment $(1,9,13-19)$. In general, hypercoagulopathy or other coagulation disorders are most often related to the development of ischemic/embolic stroke $(9,15,19)$. Cardio-embolism, large-vessel atherosclerosis and small-vessel occlusion have been reported as the major causes of ischemic stroke, while non-bacterial thrombotic endocarditis (NBTE) is rarely noted $(9,17)$. In the pathogenesis and prognosis of acute ischemic stroke, active cancer (recurrent malignant tumor, metastases, or ongoing chemo-/radiotherapy) plays an active role (1). In survivors of childhood Hodgkin's disease, who are at an increased risk for stroke, mantle radiation exposure is strongly associated with subsequent stroke and the potential mechanisms may include carotid artery disease or cardiac valvular disease (14). Nevertheless, brain tumors always remain the main etiology for stroke or other neurological pathologies, while cases where the diagnostic misinterpretation of a brain tumor as a stroke or the diagnostic misinterpretation of an underlying malignancy as an incident of CVD, are not rare (13).

Stroke in cancer patients may be hemorrhagic or ischemic $(16,20)$, while embolisms have been reported to be the most common cause of stroke (9). In a wide, population-based Swedish study, the risk [expressed as standardized incidence ratio (SIR)] of hemorrhagic and ischemic stroke in the first 6 months following cancer diagnosis was 2.2 and 1.6, respectively. While the overall stroke risk decreased rapidly with time, it remained elevated even after a decade following the cancer diagnosis (16). In that same study, metastasis was also associated with a greater risk of hemorrhagic and ischemic stroke ( $\mathrm{SIR}=2.2$ and $\mathrm{SIR}=1.5$, respectively) (16). In a similar vein, intracranial hemorrhages (ICHs) have been reported in 20 to $50 \%$ of patients with metastatic brain tumors (21). In cancer patients, cerebral infarctions have been found to be more frequent than hemorrhage $(11,22)$, whereas in patients with leukemia, hemorrhages have been found to be much more common than infarcts caused by coagulopathy or CNS infiltration $(11,16,23)$.

The present review focuses on the possible pathophysiological mechanisms and causes of stroke in cancer patients, and aims to identify the most common and specific types of stroke. Moreover, clinical manifestations are discussed, and useful modalities to diagnose the cause of stroke in cancer patients are presented, while providing valuable information on treatment and prevention measures.

\section{Cancer types associated with stroke}

To date, several studies have attempted to elucidate which cancer types present a stronger association with the occurrence of stroke. A concise presentation is presented in Table I. In a previous study, among 1,274 patients with stroke admitted to a stroke unit, $12 \%$ had an additional diagnosis of cancer, with urogenital, breast and gastrointestinal being the most frequent cancer types (15). In addition, in patients diagnosed with lung, pancreatic, colorectal, breast and prostate cancers, a higher stroke incidence was reported (24). The stroke risk also seemed to be associated with the aggressiveness of the cancer; 
Table I. Association of cancer types with ischemic (Is.S.) and hemorrhagic stroke (H.S.).

\begin{tabular}{|c|c|c|c|}
\hline Cancer type & Is.S. & H.S. & Authors/(Refs.), year \\
\hline Lung & $\checkmark$ & - & $\begin{array}{l}\text { Navi et al (24), 2015; Selvik et al (7), 2015; } \\
\text { Aarnio et al (25), 2015; Zoller et al (16), } 2012\end{array}$ \\
\hline Colorectal-GI & - & $\checkmark$ & $\begin{array}{l}\text { Stefan et al (15), 2009; Navi et al (24), 2015; } \\
\text { Selvik et al (7), 2015; Zoller et al (16), } 2012\end{array}$ \\
\hline Breast & $\checkmark$ & - & Stefan et al (15), 2009; Navi et al (24), 2015 \\
\hline Prostate & $\checkmark$ & - & Navi et al (24), 2015; Selvik et al (7), 2015 \\
\hline Pancreatic & $\checkmark$ & - & Navi et al (24), 2015; Zoller et al (16), 2012 \\
\hline Urogenital & $\checkmark$ & - & Stefan et al (15), 2009 \\
\hline Nervous system & $\checkmark$ & $\checkmark$ & Zoller et al (16), 2012 \\
\hline Skin/melanoma & $\checkmark$ & $\checkmark$ & $\begin{array}{l}\text { Zoller et al (16), 2012; Donato et al (21), 2015 } \\
\text { Dearborn et al (26), } 2014\end{array}$ \\
\hline Leukemia & $\checkmark$ & $\checkmark$ & Zoller et al (16), 2012 \\
\hline Non-Hodgkin lymphoma & $\checkmark$ & $\checkmark$ & Zoller et al (16), 2012 \\
\hline Myeloma & - & $\checkmark$ & Zoller et al (16), 2012 \\
\hline Choriocarcinoma & - & $\checkmark$ & Dearborn et al (26), 2014 \\
\hline Endocrine gland/thyroid & $\checkmark$ & $\checkmark$ & Zoller et al (16), 2012 \\
\hline Liver & - & $\checkmark$ & Zoller et al (16), 2012 \\
\hline Renal cell/kidney & - & $\checkmark$ & $\begin{array}{l}\text { Zoller et al (16), 2012; Donato et al (21), 2015'; } \\
\text { Dearborn et al (26), } 2014\end{array}$ \\
\hline
\end{tabular}

${ }^{a}$ Authors did not specify whether the stroke was ischemic or hemorrhagic; ${ }^{b}$ study was performed on patients with brain metastases.

lung, pancreatic and colorectal cancers, which presented the highest stroke risks, are usually diagnosed at a later stage than breast and prostate cancer (24). The aforementioned cancer types were also identified as the most common among patients diagnosed with cancer post-stroke (7). Lung/respiratory tract cancer had one of the strongest independent associations with death during a follow-up of patients under 49 years of age with ischemic stroke (25). Among 820,491 Swedish patients with cancer, cancers of the small intestine, pancreas, lung, nervous system and endocrine glands, and leukemia, presented a $>2$-fold higher risk of ischemic stroke in the first 6 months post-diagnosis, and this risk remained increased even after a decade after hospitalization in cancer types, such as upper respiratory/digestive tract cancer, salivary gland, colon, rectum, nose, breast, prostate, urinary bladder, skin (squamous cell), nervous system cancers and non-Hodgkin lymphoma. For hemorrhagic stroke, the cancer type pattern changed and a highly elevated risk was reported for cancers of the small intestine, liver, kidneys, nervous system, thyroid gland, endocrine glands, connective tissue, non-Hodgkin lymphoma, myeloma and leukemia (16). Similarly, patients with melanoma or renal cell carcinoma and brain metastasis were characterized by a 4-fold higher risk of ICH compared to patients with lung cancer with brain metastasis $(21,25)$. In general, melanoma, renal cell carcinoma, and choriocarcinoma are the cancer types considered to have a higher tendency for hemorrhaging (26).

\section{Pathophysiology}

Several major pathophysiological mechanisms of stroke exist in cancer patients, which can be directly related to cancer, and are caused by cancer complications, such as coagulation disorders and infections, or by therapeutic and diagnostic interventions $(4,25)$. Complications of chemotherapy, radiation therapy (RT) and hematopoietic stem cell transplantation (HSCT) for cancer can occur during the course, or even years after treatment (20). A short depiction of the various factors pertaining to a cancer-associated stroke is presented in Fig. 1.

In many cases, the establishment of whether the stroke is caused by the cancer itself and its complications or by the treatment of cancer remains a challenge. In several occasions, a combination of processes operate, and both hemorrhagic and ischemic stroke can simultaneously occur (20). Consequently, in patients with active malignancy, strokes are more frequently classified as 'of undetermined etiology' or as 'other determined etiology' by TOAST (https://radiopaedia.org/articles/toast-classification-in-acute-ischemic-stroke) classification, whereas in non-malignancy patients, the majority of strokes derives from small vessel occlusion $(19,27,28)$.

Direct tumor effects. In clinical practice, direct tumor-related stroke is rare and also difficult to identify (4). Direct tumor effects vary considerably, and include arterial and venous sinus invasion by tumor mass or leptomeningeal infiltrates, tumor emboli, blood vessel compression by tumor growth or tumor bed edema, and intratumoral hemorrhage (ITH) $(20,26)$.

Leptomeningeal metastasis, also known as meningeal carcinomatosis or neoplastic meningitis, is most commonly caused by breast cancer, lung cancer and malignant melanoma, when cancer cells spread into the cerebrospinal fluid (CSF) of the subarachnoid space (2). It is estimated to occur in 


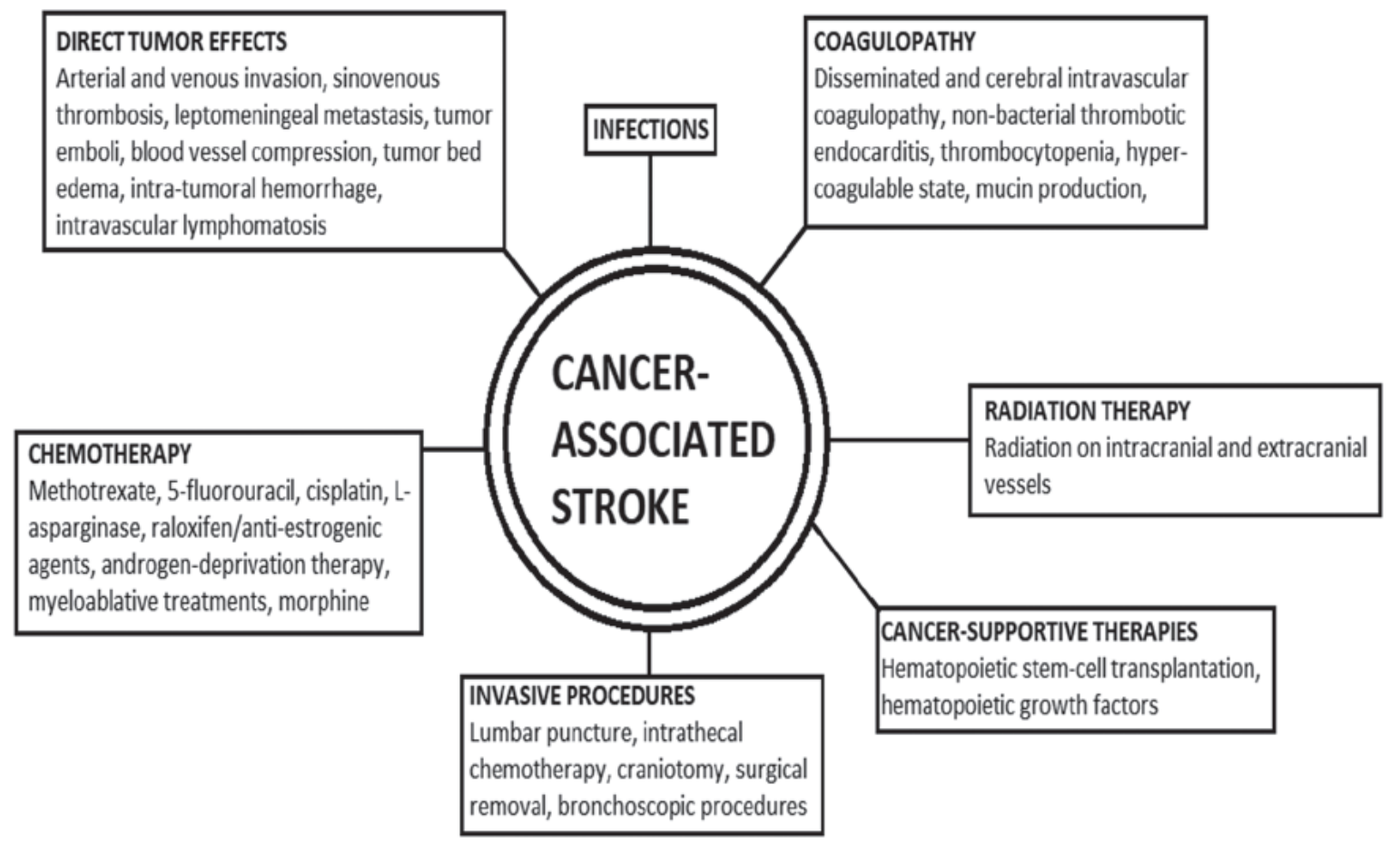

Figure 1. Schematic representation of the factors pertaining to a cancer-associated stroke.

approximately $5 \%$ of all cancer patients and is the third most common metastatic complication of the CNS (29). Multiple lesions and venous sinus occlusion from leptomeningeal carcinomatosis most commonly occur in patients with neuroblastoma, lymphoma and lung cancer $(30,31)$. These can lead to cerebral infarctions due to tumor growth in the Virchow-Robin perivascular spaces, leading to vessel thrombosis or spasm and the infiltration of vessel walls $(4,32)$.

Sinovenous thrombosis (SVT) is most widely reported in pediatric patients. Occlusion in the cerebral venous system obstructs venous outflow, leading to intracranial hypertension, which in turn may lead to cerebral ischemia (33). Risk factors for its appearance include cancer treatment, otitis media, sinusitis, trauma, dehydration, heart failure or inherited thrombophilia (34), and the superior sagittal sinus is the one most often affected $(31,35)$. In a multicenter retrospective study on patients with acute lymphoblastic leukemia (ALL), the stroke prevalence was low (at $0.47 \%$ ), but all cases were due to SVT (36). Similarly, a Nordic multicenter study reported a SVT prevalence of $2 \%$ in pediatric patients with ALL (34).

Intravascular lymphomatosis (IVL) (or malignant/neoplastic angio-endotheliosis, or Tappeiner syndrome) is a rare lymphoproliferative disorder, a form of extranodal B-cell non-Hodgkin's lymphoma, within the lumen of medium and small vessels $(4,37,38)$. Only few are of T-cell or natural killer (NK)-cell origin $(4,38)$. IVL can affect any organ, but most frequently involves the CNS, which also presents the highest rate of postmortem diagnosis and exhibits a poor survival rate, particularly in the elderly, as patients exhibit stroke-like symptoms. However, IVL is rarely included in the differential diagnosis of CNS malignancy $(37,38)$. Patients often present with diffuse encephalopathy or multifocal cerebral infarcts (4).

Arterial embolic infarction, although rare, can be the result of tumor embolization, which can be large enough to induce a transient ischemic attack (TIA) or infarction $(23,39)$. Myxoma or other heart and lung tumors can be the source of tumor emboli in the brain (4). In the literature, embolic cerebral infarcts are the most commonly observed events in left atrial myxoma patients; in 10 to $30 \%$ of whom, neurologic symptoms have been reported; these can also be the initial and sole manifestations (40). As case reports have suggested, a tumor embolus may also lead to a cerebral aneurysm via the invasion of the vessel and its subsequent dilatation, which may rupture into the parenchyma or the subarachnoid space $(4,41)$.

Cancer is also associated with hemorrhage in each brain compartment, with intraparenchymal hemorrhage (IPH) being the most frequent, followed by subdural (SDH), subarachnoid (SAH) and epidural hemorrhage (EDH), respectively (42). ITH is the most common cause of ICH in cancer patients $(42,43)$. The hemorrhage mechanism is multifactorial and includes the increased creation of dilated, thin-walled, intra-tumoral vessels, the rupture of these newly-formed vessels, the tumor invasion of pre-existent vessels and tumor necrosis (44).

Of the primary brain tumors, glioblastoma multiforme, being the most common primary brain tumor, with highly invasive cells, is most frequently linked to ICH. Oligodendrogliomas are also predisposed to hemorrhage and even benign tumors, mainly meningiomas, can be the cause of ITH (42). Solid tumors most commonly associated with ICH are lung, breast, melanoma and renal cell cancers, due to their high population incidence, brain metastases and histological components of neoangiogenesis, necrosis and blood vessel invasion. Thyroid cancer, hepatocellular carcinoma and choriocarcinoma also present an abnormally high tendency towards hemorrhage $(42,44)$. Choriocarcinoma has also been associated with the occurrence of neoplastic aneurysms (4). Granulocytic sarcomas, rare tumors that occur 
primarily in patients with acute myeloid leukaemia (AML) or other myeloproliferative disorders, may also cause ICH (45). Hematological malignancies, particularly leukemia, are also a frequent cause of $\mathrm{ICH}$, although their mechanism mainly involves disorders in coagulation (42), analyzed further below.

$\mathrm{SDH}$ in cancer patients is usually the result of coagulopathy or trauma, alongside dural metastases $(23,42,46)$. The occurrence of SDH is speculated to be the result of the rupture of vessels within the metastatic tumor, the erosion of adjacent vessels by the tumor, or the rupture of the inner dural vessels due to congestion of the outer vessels (23). However, only $15-40 \%$ of patients with dural metastases have a coexistent SDH (42). SDH has been most commonly reported in leukemia, prostate, lung and breast cancer and lymphoma (46). SAH and intraventricular hemorrhage are also usually due to coagulopathy, trauma or ITH, and very rarely from arteriovenous malformations. When aneurysmal SAHs occur in cancer patients, they should be investigated for atypical etiologies, such as mycotic or neoplastic. These aneurysms are fusiform, typically develop in distal middle cerebral artery branches and are commonly tied to atrial myxoma, choriocarcinoma and lung carcinoma (42). Finally, EDH in association with a skull metastasis is a rare incident (47).

Hyperleukocytosis, defined as a white blood cell (WBC) count of $>100,000$ per $\mathrm{mm} 3$, has been commonly reported in acute leukemia, causing CNS leukostasis $(48,49)$. The accumulation of leukemic blast cells within the capillary vascular lumen can result in ICH (49) and mainly medium-sized vessels are involved (50). It occurs in approximately $7 \%$ of pediatric patients with acute leukemia, worsening its prognosis as it is associated with a higher mortality rate (49). Finally, in multiple myeloma patients, ischemic stroke can be the result of hyperviscosity syndrome due to elevated protein levels (4).

Coagulopathy. Coagulopathy is one of the main causes of stroke in cancer patients. Coagulation disorders are the most common cause of CVD in cancer patients and involve disseminated intravascular coagulopathy (DIC), NBTE and thrombocytopenia $(10,20)$. The first post-mortem characteristics of cerebral intravascular coagulation were described in 1975, in patients with breast cancer, leukemia and lymphoma, in the setting of widespread metastasis and sepsis (51).

In patients with cancer, the most common cause of cerebrovascular thrombosis (CVT), identified in several clinical series, is a hypercoagulable state that accompanies cancer, resulting in systemic and cerebral arterial or venous thrombosis $(19,52)$. In intravascular coagulation, tumor procoagulant activity, host inflammatory responses and extrinsic factors are involved. Tumor cells express the procoagulants, tissue factor (binds to factor VII) and cancer procoagulant, and release inflammatory cytokines and vascular endothelial growth factor, mediators that enhance procoagulant activity and angiogenesis $(6,26,53)$. They also overexpress cytokines that attract leucocytes, possibly triggering an inflammatory response, with prothrombotic effects (16). Lung and pancreatic cancers are the most common cancer types causing this coagulopathy $(24,52)$.

In 2015, an analysis by Karlińska et al stated that stroke patients with an active cancer tend to demonstrate lower hematocrit levels, higher serum C-reactive protein (CRP) levels, and a higher erythrocyte sedimentation rate when compared to cancer-free stroke patients. Since it is known that the aforementioned inflammatory markers are associated with coagulation, these findings indicate that, indeed, in active malignancy, the cancer-specific prothrombotic mechanisms may play an important role in stroke pathophysiology (27).

Specifically concerning adenocarcinomas, Dearborn et al (2014) reported that they are considered to potentiate thrombi via the production of mucin, a high molecular weight molecule that is glycosylated and secreted normally by endothelial cells. Adenocarcinomas, particularly those of the pancreas, colon, breast, lung, prostate and ovarian system, can secrete this molecule directly into the bloodstream, assisting in the appearance of a viscous and hypercoagulable state. Mucin can also interact with certain cell adhesion molecules on endothelial cells, platelets, and lymphocytes to induce the formation of platelet-rich microthrombi (26).

A broad small arterial and venous thrombotic vasculopathy can also manifest in cancer patients (13). It is termed 'cerebral intravascular coagulopathy' when the predominant signs are neurological, and is considered as the CNS equivalent of cancer patients' systemic thrombotic microangiopathy (35). This condition is not often reported (35), and it is speculated to be more common than originally estimated, as it can be mistaken with other encephalopathy causes in cancer patients, and its diagnosis can only be confirmed at autopsy (23). In the first autopsy study by Graus et al in 1985, it was only reported in $8 \%$ out of 500 patients (11)

Depending on the type, cancer can also be linked to acute or chronic DIC. In DIC, a disruption in the balance between thrombus formation and thrombolysis can either lead to the thrombotic occlusion of vessels, due to the excess activation of the coagulation process, or to diffuse hemorrhage, due to the subsequent depletion of platelets and coagulation factors. Therefore, in cancer patients, it can manifest as thrombotic stroke and intracerebral hemorrhage (4). A variety of thrombohemorrhagic entities, such as low-grade DIC, are associated with solid tumors, whereas leukemia, myelocytic and lymphocytic, usually present acute DIC, which manifest as marked bleeding (4,54). Patients with pancreatic cancer and adenocarcinoma have particularly been reported to be at a very high risk of DIC (55). Acute promyelocytic leukemia is strongly associated with severe hemorrhage in the setting of DIC and other hemostatic disorders (54). These bleeding manifestations usually occur in the parenchyma or the subdural compartment, and rarely in the subarachnoid space $(4,56,57)$.

NBTE, also known as marantic endocarditis, is defined as non-infectious, sterile cardiac valvular vegetations with negative blood cultures, and it is most commonly caused by an underlying malignancy (58). The cancer in this case is more often widespread and cerebral infarction is a late complication; however, in rare instances, NBTE with cerebral infarction is the presenting sign of cancer (59). In NBTE, sterile platelet-thrombin vegetations develop on cardiac valves (almost exclusively the left heart valves; mitral and aortic) in association with widespread systemic and cerebral thrombosis $(60,61)$. It usually presents with systemic and pulmonary embolization, with the most common neurological complication being ischemic stroke (58).

The exact NBTE prevalence in cancer patients has not been accurately estimated, as tissue diagnosis is often inaccessible 
and echocardiographic studies are not widely used to screen cancer patients for NBTE (62). Some original autopsy studies have reported NBTE in $9.3 \%$ of cancer patients, and cancer in $59 \%$ of patients with NBTE $(63,64)$. In a study by Edoute et al (1997), valvular vegetations consistent with NBTE were found in $19 \%$ out of 200 cancer patients (65); Liu and Frishman (2016), several years later, also reported NBTE in $19 \%$ of disseminated adenocarcinomas (61). Of note, Taccone et al (2008) reported NBTE as the most common cause of stroke in their cancer patients, followed by intravascular coagulation and atherosclerosis (5). As an entity, NBTE is most commonly reported in adenocarcinomas, particularly mucin-producing carcinomas of the lung or the gastrointestinal tract, lymphoma, and also in carcinomas of the ovarian, the pancreas and biliary system $(35,61,62)$. Adenocarcinomas, as previously mentioned, are considered to potentiate thrombi via the production of mucin (26).

Thrombocytopenia may be the result of hematological cancers or, most frequently, the result of chemotherapy $(54,57)$. Additional causes of significant thrombocytopenia include tumor involvement of bone marrow and spleen, microangiopathic disorders such as DIC, thrombotic thrombopenic purpura (TTP) or hemolytic uremic syndrome (HUS). Lymphoproliferative malignancies can also be associated with secondary immune thrombocytopenia (54). In a study by Correale et al (1990) on 989 patients with lymphoma, 2\% had $\mathrm{ICH}$, in clear association with platelet alterations (66) and in a 2013 study on pediatric cancer patients, all those suffering with ICH had platelet counts of $<100,000 / \mathrm{mm}^{3}$ (67).

Infections. Infections rank among the first causes of morbidity in cancer patients, particularly in those with hematological malignancies, where autopsy studies have demonstrate that approximately $60 \%$ of deaths are infection-related (68). Cancer patients are frequently immunocompromised, and thus are automatically at risk of infection, relevant to the type of cancer $(4,68,69)$, and the association between stroke and systemic infection has been well established. The organisms most frequently linked to an increased risk of stroke include Helicobacter pylori, Chlamydia pneumoniae, Mycoplasma pneumoniae, Haemophilus influenzae, Epstein-Barr virus, herpes simplex virus (HSV)-1 and HSV-2, and cytomegalovirus (CMV) (70).

The systemic inflammatory response induced by the pathogen can damage the vascular endothelium and predispose patients to ICH (70). Infectious endocarditis is a predominant cause of stroke, via embolism to the brain. The degradation of the arterial wall by bacteria or septic emboli results in abnormal dilatation or mycotic aneurysms (70). These aneurysms usually occur at distal branches of the middle cerebral artery and can rupture, leading to ICH $(56,70)$. Staphylococcus aureus, $\beta$-hemolytic streptococci and Streptococcus viridans are the bacteria which most commonly complicate an infective endocarditis with $\mathrm{ICH}(70)$.

Moving on to more specific mechanisms, HSV-1 meningoenchephalitis can lead to petechial cerebral hemorrhages and $\mathrm{ICH}$ in its severe forms. Syphilis causes an obliterative endarteritis, which may in turn lead to ischemic stroke through progressive luminal stenosis. Tuberculosis (TB) can present infarcts in tuberculous meningitis, which accompanies pulmonary $\mathrm{TB}$ in $1 \%$ of the patients (70). In cancer patients, opportunistic infections with pathogens, such as the JC40 virus can cause a progressive multifocal leukoencephalopathy, which maybe be mistakenly characterized as a cerebrovascular lesion (4).

Fungal infections are the second most common in cancer patients, following bacterial ones (68). Fungal meningitis can be caused by yeasts (such as Cryptococcus spp. and Candida spp.) and moulds (such as Aspergillus spp.) (70). Yeasts can cause stroke, ischemic or hemorrhagic, via a vasculopathy of the large vessels traversing the subarachnoid space, venous outflow obstruction, endarteritis and the formation of abscesses linked to hemorrhage. They do not usually invade cerebral vessels; moulds on the contrary, produce enzymes that allow vessel-wall invasion, causing mycotic arteritis or aneurysms (70). The hematogenous spread of septic emboli to the brain can trigger an ischemic or hemorrhagic stroke, with or without the presence of arteritis and aneurysms $(20,70)$. Cerebral Aspergillus infections are mostly secondary to lung infection, whereas Candida ones usually originate from the gastrointestinal or genitourinary tract (4). Fungal septic emboli can also occur in leukemic patients who have undergone bone marrow transplantation (23).

Viral infections are not a rare instance either, in cancer patients, and are usually the result of reactivation of a latent disease, primarily in hematological malignancies. Parasitic and unusual infections should also be considered in patients with a history of exposure (68).

Susceptibility to infections in cancer patients is due to host-associated and treatment-related factors $(68,69)$. The former include underlying immune deficiencies, comorbidities, ulcerating lesions in mucosal surfaces, past infections, poor nutritional status, catatonic state and psychological stress, while the latter consist of surgery and invasive procedures, radiation, immunosuppressant regimens and antimicrobial use $(68,69)$. However, the identification of a sole immunodeficiency factor in cancer patients is utopic in common clinical practice, as multiple deficiencies usually co-exist (68).

Hematological malignancies heavily burden patients in terms of immunodeficiency. Patients with acute leukemia and lymphoma manifest neutropenia, due to the disease itself or the cytotoxic chemotherapy, and present different types of infections (68). Typically, Gram-negative bacilli, such as Escherichia coli, Klebsiella spp. and Pseudomonas aerugi$n o s a$, are the causes of the earliest infections. However, an increase in infections caused by Gram-positive aerobic bacteria, mainly staphylococci and streptococci, has recently been reported $(68,69)$. Fungal and viral infections occur later in the course of neutropenia (68), and patients with neutropenia are particularly prone to developing bloodstream infections (BSIs), particularly following bone marrow transplantation (69). On the other hand, chronic lymphocytic leukemia (CLL) usually leads to deficits in humoral immunity, while additional defects in cell-mediated immunity, complement activity, and neutrophil and other phagocytic cell activity manifest following the treatment. Hypogammaglobulinemia predisposes patients to infections by Streptococcus pneumoniae, Haemophilus influenzae, Neisseria meningitidis and Escherichia coli, whereas treatment modalities, such as alkylating agents predispose to streptococcal, staphylococcal, and enteric Gram-negative 
bacterial infections. Additionally, purine analogs or alemtuzumab treatments predispose to opportunistic infections with Listeria spp., Mycobacterium tuberculosis, Nocardia spp., Candida spp., Aspergillus spp., Pneumocystis jiroveci and herpesviruses. Finally, multiple myeloma increases the susceptibility to infections with encapsulated bacteria such as Streptococcus pneumoniae, Haemophilus influenzae and Neisseria meningitidis (68).

Solid organ tumors do not lead to the same degree of immunodeficiency as hematological malignancies, mainly as chemotherapy in these cases does not cause long-term or profound neutropenia; a few exceptions exist, such as metastatic breast carcinoma, prostate, lung, adrenal, thyroid and kidney cancers, that tend to infiltrate the bone marrow and actually cause neutropenia in advanced stages (68). Any tumor that disrupts anatomical barriers can give way to infections, and specifically skin cancers are usually associated with staphylococci and streptococci, oral cavity and nasopharynx with anaerobic bacteria, streptococci and Haemophilus influenzae, gastrointestinal tract with enterobacteriaceae and fungi, and the female genital tract with Enterobacteriaceae, anaerobic Gram-negative bacteria, Enterococci and Clostridium spp. (68). In a study using predominantly solid-organ-cancer patients to examine the microorganisms that cause BSIs, Escherichia coli was the most common Gram-negative bacterium cultivated in the cancer patient group. Cancer patients also presented twice as many BSIs by Enterococcus faecalis, Enterococcus faecium, Pseudomonas aeruginosa and Enterobacter cloacae. Doubled was also the percentage of positive yeast blood cultures from cancer patients compared with non-cancer patients (69).

Chemotherapy. Chemotherapy has often been blamed as the cause of cerebral arterial or venous thrombosis, although this can occur in the setting of advanced malignancy as well (56). Chemotherapy can lead to stroke via endothelial toxicity and abnormalities in coagulation and hemostasis factors (71). It can also trigger the manifestation of a stroke by transferring susceptibility via immunosuppression and the increase in opportunistic infections (68). In general, the risk of a chemotherapy-induced stroke is rather low and the risk is higher for some specific regimens, such as methotrexate (MTX), 5-fluorouracil, cisplatin and L-asparaginase (4,71). However, even neoadjuvant chemotherapy (NC) has been linked to an increased risk of stroke; Abt et al (2014) reported that NC was linked to a higher risk of short-term stroke and mortality in patients undergoing brain tumor resection (72).

L-asparaginase has one of the best known associations with thrombosis and stroke $(56,71,73)$. It is typically used in combination regimens for breast cancer and in the induction therapy of ALL $(4,56)$. It has repeatedly been associated with CVT in children treated for leukemia $(4,26)$, as asparaginase and steroids are deemed strong prothrombotic agents (34). The cerebrovascular effects can manifest as cerebral thrombosis or hemorrhage, and the patients that receive such treatments should be closely monitored and the treatment should be interrupted in the case of a cerebrovascular event (CVE) (4).

All agents with anti-estrogenic effects may increase the risk of CVD (74,75). More specifically, raloxifene, a selective estrogen receptor modulator, has been linked to a higher incidence of stroke and thromboembolic events (76).
For tamoxifen, historically the standard endocrine therapy for breast cancer, the reported findings vary among studies. It was the only factor associated with an increased risk of stroke in 4,414 10-year survivors of early breast cancer (77) and was associated with a significantly increased risk of venous thromboembolic events, pulmonary embolism and stroke, as reviewed by Esteva and Hortobagyi (74). In other analyses however, tamoxifen alone was not associated with an increased risk of CVD (78), but only when combined with hypertension (79). Using data from the Swedish National Hospital Discharge Registry and the Swedish Cause of Death Registry, the incidence of CVD was increased during the active treatment phase and decreased in the post-treatment period (80). Contrary to the above, a Taiwanese study including 3,690 breast-cancer women revealed a significant reduction in cardiovascular events, including hemorrhagic and ischemic stroke (81). Hence, the literature data on tamoxifen have yielded conflicting results (4).

In three large-scale studies, patients with prostate cancer treated with endocrine hormonal therapy [androgen deprivation therapy (ADT)] were found to be at an increased risk of stroke $(75,82,83)$. In a smaller prospective study from Taiwan, no significant difference was found in the risk of stroke between ethnic Chinese patients with prostate cancer who did or did not receive ADT, after adjusting for potential confounders (84). A review of the literature by Meng et al (2016) concluded that ADT does indeed present an increased risk of stroke. Significance was reached when ADT monotherapy was examined after removing prostatectomy and radiotherapy patients, and in gonadotropin-releasing hormone (GnRH), GnRH plus oral antiandrogen and orchiectomy treatments (85).

Platinum compounds, clinically, seem to present the highest risk of stroke (4). Cisplatin has repeatedly been reported to be associated with CVEs $(4,71,86)$. However, the mechanisms involved remain largely unknown. Circulating endothelial- and platelet-derived particles can contribute to cisplatin-induced stroke (4). Kuan et al (2014) reported an elevated relative risk (RR) of stroke in patients with ovarian cancer that were treated with cisplatin-based or carboplatin-based chemotherapy agents, as opposed to non-platinum-based regimens (87).

Stroke-like events and stroke have been reported in MTX treatment $(4,88)$. In pediatric patients, they have been linked with acute treatment, but not with subsequent administration (88). However, long-term survivors from pediatric cancer groups were found to be 40 -fold more prone to developing stroke than their sibling controls (4).

Brain hemorrhages can also manifest in the setting of chemotherapy treatment. The chemotherapy of AML with a monoblastic component has been associated with a high incidence of SDH (89); an associated hemorrhagic vasculitis or cerebritis has occasionally been reported as well (56) and hemolysis from chemotherapy administration is a rare cause of brain hemorrhage (23). Chemotherapy, particularly the myeloablative treatment of hematological malignancies, remains the most common cause of thrombocytopenia in cancer patients. However, several non-myeloablative chemotherapeutic agents have also been connected to its appearance, even though most of the classic chemotherapeutic treatment plans in cancers, such as of the colon, lung, breast and prostate, have a low incidence of National Cancer Institute (NCI) 
Grade 3 (platelet count of 25 to $\left.49.9 \times 10^{9} / 1\right)$ and $4\left(<25 \times 10^{9} / 1\right)$ thrombocytopenia. Moreover, thrombocytopenia development has also been related to newer therapeutic agents. When examining patients treated with sunitinib, a multi-targeted tyrosine kinase FDA approved for the treatment of renal cell carcinoma, pancreatic neuroendocrine tumors and gastrointestinal stromal tumors, $7.6 \%$ of them presented high-grade (NCI Grade 3 or 4) thrombocytopenia (54).

Finally, a recent study based on a large malignancy cohort of cancer patients, demonstrated that symptomatic therapy in cancer patients may be related to stroke as well; intense morphine treatment is associated with an increased stroke incidence in cancer patients, and the association is particularly significant for prostate cancer patients (90).

Radiation therapy. RT has been shown to be an independent risk factor for cardiovascular disease and CVD in cancer patients (91-93). Post-radiation vasculopathy occurs in intracranial and extracranial vessels, with medium and large-sized vessels being most frequently affected (4). The subsequent stenosis or occlusion is typically more extensive within the radiation portal, than the atherosclerosis which develops in its absence as well (56).

Radiation to the neck has been related to subsequent vascular wall thickening, atherosclerotic plaque formation and vascular damage $(26,91,94)$. Studies have also confirmed that RT predisposes to the formation of inflammatory plaques, which are more likely to rupture and cause a stroke, or a heart attack (91). Patients that have received RT to the head and neck area have also been shown to have significant internal carotid artery/common carotid artery stenosis (95), and notably, patients can develop a radiological image that closely resembles Moyamoya syndrome; stenosis of the carotid vessel with abnormal netlike vessels and transdural anastomosis distal to the stenosis (26). The frequency of secondary-to-RT internal carotid stenosis ranges from 12 to $60 \%$, depending on the study (4). Thus, several studies using different methods and patients with varied disease processes have reported an increased risk of CVEs following RT for head and neck cancers or lymphoma $(12,92,94,96,97)$. The first large study was conducted in 1981 and demonstrated a post-cervical-RT stroke incidence of $6.3 \%$ (96). Subsequent studies further reported a 15 -year cumulative stroke risk of $12 \%$ following $\mathrm{RT}$, and RT to the neck and mediastinum to be an independent risk factor for CVD $(12,97)$. Scott et al (2009) reported a crude stroke risk of $2.6 \%$ following neck RT, compared to $0.29 \%$ of non-RT patients (94), whereas Smith et al (2008) associated an excess CVD risk to definitive RT for head and neck cancers, but not to post-operative RT in older patients (98). Concerning the effects of RT on operated patients, a 2014 study compared patients with lung cancer, and found a higher stroke incidence (almost double) compared to post-operative RT and with surgery alone and a lower two-year-stroke-free survival rate (99). However, finally, radiation fields that included the carotid artery did not seem to increase the risk of stroke in breast cancer survivors in three large-scale studies $(6,79,100)$.

Pediatric cancer patients are not excluded from this side-effect; their cerebral vessels can also be the subject of stenosis or occlusion following RT. Morris et al (2009) described the manifestations of RT-induced CVD in pediatric patients having received RT to the brain and/or the neck, and they include steno-occlusive disease, aneurysm, mineralizing microangiopathy, vascular malformations and stroke-like migraines (101). A 2005 study found a RR for late-occurring stroke in patients who received mantle RT of 5.62 (14). Mueller et al (2013) confirmed that cranial irradiation placed childhood cancer survivors at a risk of both, first and recurrent, and stroke (102). Finally, a large cohort study revealed that the risk of stroke in childhood cancer survivors was associated with cranial RT in a dose-dependent manner (103).

Cancer supportive therapies: HSCT and hematopoietic growth factors (HGFs). HSCT and HGFs are increasingly used in cancer care in support of standard anticancer therapies in order to limit bone marrow toxicity due to chemotherapeutic agents and to ameliorate the quality of life of patients, reducing asthenia, thrombocytopenia and neutropenia. Both HSCT and HGFs are considered cancer-supporting therapies; however, there are still concerns about the safety of these treatments, particularly regarding peripheral and cerebral cardiovascular complications (20). To date, several mechanisms have been described for HSCT-related hemorrhagic stroke, including graft-versus-host disease (GVHD) (only for allogenic transplantation), alterations in coagulation profiles and infections $(35,104,105)$. Syed et al, in 2016 (105), reported that cerebral IPH occurred after a median time of 122 days after HSCT in 1.1 to $2.4 \%$ of the patients with a higher mortality rate compared to subarachnoid hemorrhage or subdural hematoma $(105,106)$. Furthermore, another cerebrovascular complication related to HSCT is that of vasculitis, which rarely occurs due to chronic GVHD, leading to cerebral infarction, hemorrhage, or leukoencephalopathy (106-108).

Cerebrovascular complications following HSCT are potentially fatal events. Generally, cerebrovascular hemorrhagic events are related to recalcitrance to platelet transfusions, to arterial hypertensions, low fibrinogen serum levels and, as previously described, to high-grade GVHD (109). Usually, the onset of cerebrovascular complications is characterized by worsening sensorium and headache without lateralizing signs. However, in some cases, the onset of CVDs is asymptomatic, making a firm diagnosis difficult (110). The diagnosis of stroke and other CVDs is often performed by a computer-assisted tomography (CT) scan, as this technique allows for the highlighting of the hemorrhagic regions as hyper-intense areas assisting the diagnosis of intracranial bleeding. The CT scan is preferred as this reveals possible lesions at least $12 \mathrm{~h}$ earlier than what can be done with magnetic resonance imaging (MRI), which is therefore not widely used. However, sometimes patients are asymptomatic with negative $\mathrm{CT}$ scans in $20-25 \%$ of patients $(109,111,112)$.

Overall, conflicting data have been generated as regards the frequency of CVDs and hemorrhagic stroke following HSCT. Katz and Segal (2005) reported a hemorrhagic stroke rate of $>32 \%$ established following the autopsy examination of patients who died as a result of HSCT (20). Liu et al (2017) recently presented the results of an observational study conducted on 459 adult patients undergoing allogeneic HSCT at an Asian tertiary medical center between January, 2003 and December, 2015. Those authors reported that the percentage of both ischemic and hemorrhagic stroke occurred only in $5.2 \%$ of 
HSCT-treated patients (24 out of 459) (113). Zhang et al (2016) studied the risk of ICH in a cohort of 2,169 patients treated with HSCT for both malignant and non-malignant hematopoietic disorders. These investigators reported that only $1.5 \%$ of these patients ( 32 patients) developed an ICH complication in a median onset time of 147.5 days (114). These data underline that the risk assessment of HSCT-related stroke is not yet completely defined, probably due to different pathological manifestations that characterize CVDs, among which the most common are IPH, SDH, SAH and multiple hemorrhage lesions in the brain parenchyma. Hence, there is a need for a better understanding of the prodromal symptoms of ICH after HSCT in cancer patients with hematological and non-hematological malignancies.

As described above, numerous studies have associated the onset of stroke with the use of hematopoietic stem cells for the treatment of oncohematological diseases, while regarding the existing association between stroke and colony-stimulating factors, current data is still limited and conflicting.

Over the past decades, patients diagnosed with cancer have begun to be treated with HGFs, which consist of colony-stimulating factors (CSFs) and erythropoiesis-stimulating agents (ESAs). Both CSFs and ESAs are commonly used to prevent infection and neutropenia in patients receiving chemotherapy and for the treatment of asthenia by stimulating the growth of red blood cells, respectively (115-117). The use of CSFs and ESAs results in better cancer patient adherence to chemotherapy without a reduction in dose administration, thus improving the clinical outcomes of the therapy $(118,119)$.

The use of CSFs and ESAs has definitely improved the effectiveness of chemotherapy treatments, although several observational and randomized studies have indicated that these HGFs had some short- and long-term side effects in several cancer pathologies $(120,121)$. Among these side-effects are venous thromboembolism (VTE), stroke, ischemic heart disease and AML or myelodysplastic syndrome (MDS) in patients with various types of cancer, including breast cancer (120-122).

Several studies have assessed the risk of AML or MDS following the administration of CSFs and ESAs in cancer patients. However, there are still doubts concerning the safety of these treatments and their potential increased risk of developing vascular and cardiovascular diseases (e.g., stroke and VTE) $(123,124)$. In particular, the use of CSFs and ESAs has been related to the development of blood clots and vascular dysfunctions, including stroke (125). For these reasons, in 2007, the Food and Drug Administration (FDA) stated that the use of HGFs can be dangerous and therefore, the safety of these compounds still needs to be verified (126).

Du and Zhang (2015) and Du et al (2016) evaluated the association between the use of CSFs and ESAs and the increased risk of VTE, stroke, ischemic heart disease and AML/MDS onset in patients with breast and colorectal cancers $(120,121)$. These authors demonstrated that patients with colorectal cancer treated with chemotherapy combined with CSFs and ESAs had an increased risk of MDS, VTE and to a lesser extent, an increased risk of ischemic heart disease (120). With regards to breast cancer patients, the retrospective cohort study by Du et al demonstrated that the combination of chemotherapy, CSFs and ESAs was associated with a 2-fold increased risk of developing VTE (2.01 hazard ratio) and AML/MDS (4.55 hazard ratio) and a weak increased risk of ischemic heart disease (1.08 hazard ratio), while the use of both CSFs and ESAs was not associated with an increased risk of stroke (121). These two studies demonstrated that the use of HGFs was not associated with the risk of stroke in cancer patients. Other studies have also tried to define the risk associated with stroke following the administration of CSFs and ESAs; however, studies are limited in this regard with inconsistent data, preventing the proper estimation of the real risk of stroke events following the administration of HGFs as supportive therapies in cancer patients (127-130).

Finally, a growing body of evidence has indicated how the use of granulocyte colony-stimulating factor (G-CSF) and granulocyte macrophage colony-stimulating factor (GM-CSF) may induce the recovery of a stroke-related cerebral damage via neuroprotective and neurorepairing mechanisms involving the activation of bone marrow-derived CD34(+) stem cells and the reduction of the lesion volume (131-133). In particular, the administration of G-CSF appears to be safe and well tolerated by patients, although further studies are required to verify the efficacy and tolerability of these factors for the treatment of stroke.

Invasive procedures. Cancer patients are subjected to a wide variety of aggressive treatments and tests, including invasive procedures, many of which can induce stroke. Cancer can heavily impact the surgical outcome as well. As Jacob and Kostev (2016) have previously suggested, cancer has a negative effect on the occurrence of intraoperative and post-procedural complications, with colorectal and breast cancers presenting the strongest association (134). Specifically, lumbar puncture, intrathecal chemotherapy and craniotomy have been linked to an increased risk of SDH $(42,46)$. In surgery, the risk of an embolic stroke is generally elevated, as it may promote the release of emboli. Pulmonary interventions in particular, such as bronchoscopic biopsies and lung surgery, have presented stroke peri- and post-operatively (4).

In a study carried out on patients with glioma with ischemic stroke, Kamiya-Matsuoka et al (2015) (135), reported that $53 \%$ of the patients had post-operative stroke and 33\% had a CVE after 2 weeks of surgery; the episodes were, therefore, quite frequent in the post-operative period. The majority of the stroke cases were close to the resection cavity, possibly suggesting iatrogenic causes. However, surgery in general remains a main stroke risk factor in these patients. Further adding to the aforementioned data, the events were frequent, particularly in patients treated with prior chemotherapy and radiation (135).

\section{From stroke to cancer}

Despite a plethora of evidence in the literature linking the occurrence of stroke to malignancy, the retrograde association between cancer and stroke has yet to be proven. It remains to be determined as to whether stroke causes cancer or whether it is likely an early manifestation of cancer $(136,137)$. A 2017 survey of Taiwanese patients found out that although patients with stroke presented a lower risk of developing any type of cancer compared to the controls, patients with brain cancer 
presented a higher risk (adjusted RR of 3.09). In the same survey, 40-60 years old females had a higher risk of developing brain cancer, while overall, the mean time for developing any type of cancer, including brain cancer, was significantly shorter in the stroke group. The researchers also reported that upon examining their own cases of malignant gliomas, only the ones with stroke previous to cancer were strongly histologically stained for HIF-1 $\alpha$, a key hypoxic regulator, possibly signifying the connection between stroke and the malignancy (136).

Similarly, but possibly showing somewhat conflicting results by gender stratification, a study on menopausal women found an overall lower incidence of cancer in women with a history of stroke compared to women without stroke and adjusted for covariate factors (137). However, it is speculated that the observed lower incidence of cancer in women that suffered a stroke and survived long enough to develop a cancer, could have been associated with lifestyle changes occurring following the survival of the stroke (137).

\section{Clinical presentation and diagnosis}

As stroke greatly affects the prognosis of a cancer patient, its early discovery remains critical. The interval time from cancer diagnosis to stroke manifestation varies considerably and depends on the cancer type as well; solid tumors usually take longer periods of time than hematological malignancies (31). When a stroke occurs in a cancer patient, the clinician can/must pinpoint its cause by carefully examining the clinical setting, taking into consideration the cancer type and its treatment (11). However, caution is advised, as an increase in brain tumors has been noted within the first year after stroke, insinuating that brain tumors can be misinterpreted as strokes (13). The original autopsy study by Graus et al (1985) described the main clinical presentation of CVD in cancer patients to resemble that of a diffuse encephalopathy more than the typical image of acute onset with a focal deficit (11).

ICH commonly presents with focal neurological deficits, headaches and encephalopathy; the presentation in cancer patients largely follows the general population. Other common symptoms include hemiparesis, nausea or vomiting, seizure and maybe even coma. Occasionally, symptoms may be gradual and non-specific, largely characterized by confusion and lethargy, particularly in SDH (42). Primary or metastatic hemorrhage in the brain or dura may also produce the initial clinical signs of a brain tumor or a change in chronic signs induced by the tumor (56). In gliomas, hemorrhages appear within the tumor, while in the majority of metastatic brain tumors, they are located around the tumor borders (44). The initial diagnostic evaluation of these hemorrhages does not differentiate from the standard guidelines (42). In the case of a suspected $\mathrm{ICH}$, patients should first be evaluated with a non-contrast head CT and if there are no contraindications, post-contrast sequences and CT angiography can further assist in locating an underlying tumor or vascular malformations (42).

Cerebral metastasis can occasionally be the initial cancer manifestation and patients may develop intracerebral hemorrhage with a stroke-like image (138). Brain metastases can present with a wide variety of neurological symptoms, with the most common ones being headaches, an impaired mental status and focal weakness, with usually a hemiparetic pattern. Sensory loss and gait deficits typically involve one side of the body, as the tumor affects its respective hemisphere (29). Metastatic brain tumors can be confirmed by a CT scan or MRI, where they typically appear rounded, well-circumscribed, non-infiltrative, with an excessive amount of edema and almost always enhanced by contrast mediums. In some cases, a biopsy is needed to reach a firm diagnosis (29).

Leptomeningeal metastases should be considered in cancer patients with signs and symptoms involving more than one anatomic site within the nervous system. The spine is affected in the majority of leptomeningeal carcinomatosis patients; the most common manifestations include neck or back pain, asymmetric reflexes, extremity weakness (usually affects both legs), pain, spinal tenderness and cranial nerve palsies, that appear in more than half of the patients (29). Rarely, as case reports have suggested, it can present with signs of meningitis and focal cerebral infarction (32). The staging of leptomeningeal metastasis includes contrast-enhanced brain and spine MRI, along with a radionuclide CSF flow study (29). Leptomeningeal dissemination has also been shown, in a case report, to resemble cerebral vasculopathy (30).

Cerebral venous occlusion often presents with headaches in cancer patients $(31,51)$. In children, symptoms and signs are age-related, as seizures and an altered mental status are the most frequent manifestations in newborns, as opposed to headaches, vomiting, lethargy and sixth cranial nerve palsy in children and adolescents. The findings of a physical examination may only include the altered mental status, or even signs of intracranial hypertension, such as papilledema (33). Tumor-related CVT usually develops gradually and can also produce signs of elevated intracranial pressure (ICP) (56). Combining the results of two studies on patients with pediatric ALL and SVT (2005 and 2015), the most common manifestations are diffuse neurological signs, seizures, headache, fatigue, cerebral nerve palsies and hemiparesis $(34,36)$. CT, MRI and the respective venographies can be used to diagnose venous occlusion $(31,34)$. The 'empty delta' sign in a CT scan represents a lack of enhancement in the thrombosed sinus and is considered characteristic $(4,30)$. Simultaneously, a tumor or associated occurrences such as infarction, hemorrhage and infiltrates can also be detected (20,30).

Intravascular lymphomatosis (IVL) can also occur with stroke-like symptoms (38) and in general, the signs and symptoms are related to the involved organs (139). MRI has limited use in its diagnosis, as it does not present enhancement and the majority of diagnoses are still made post-mortem; an unfortunate realisation, since IVL is sensitive to chemotherapy when diagnosed (38). Specifically, it is accompanied by many false negative results as it typically arises without tumor masses or lymphadenopathy (139). Patients usually display a pathological hemogram, with anemia in more than half of the patients, and increased lactate dehydrogenase (LDH) and $\beta 2$-microglobulin levels in the vast majority. Organ-specific symptoms require specific laboratory or imaging exams, and eventually biopic assessment (139).

The course of intravascular coagulopathy is progressive and can result in coma and death (51). These progressive and extensive vessel occlusions can lead to diffuse microinfarctions and encephalopathy, with superimposed transient focal signs, 


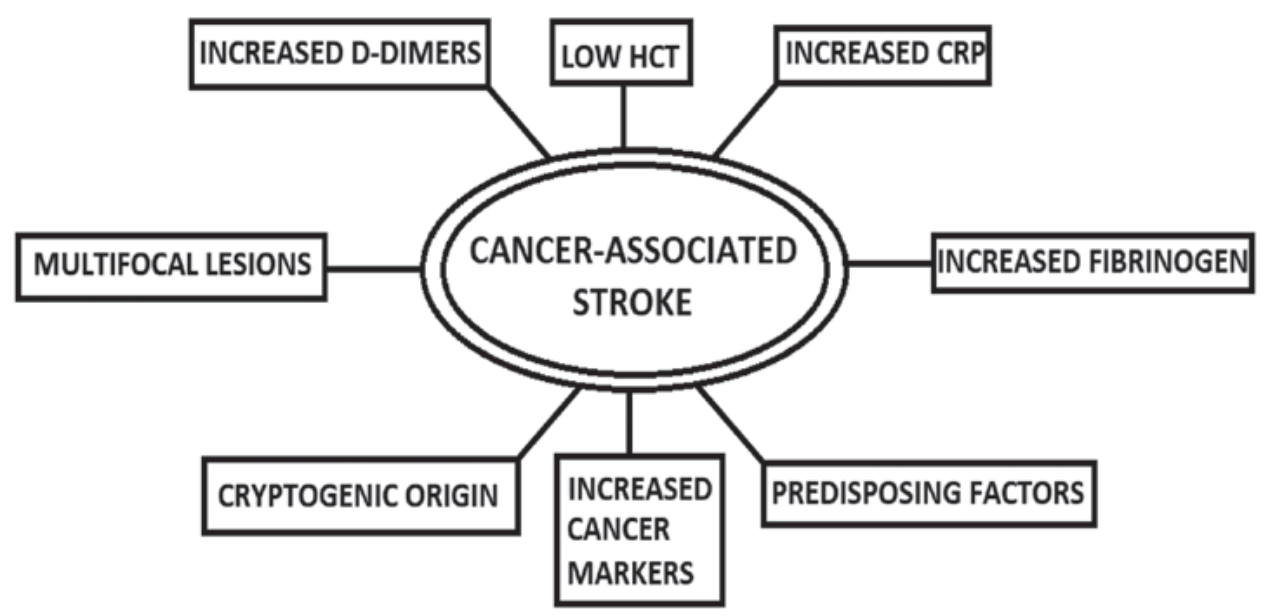

Figure 2. Factors hinting towards a cancer-associated stroke. HCT, hematocrit; CRP, C-reactive protein.

such as partial focal seizures (23). In leukemia and lymphoma, the coagulopathy is typically that of acute DIC and can lead to systemic and brain hemorrhages $(4,54)$, mostly located in the brain parenchyma or the subdural compartment (4). Laboratory results should be interpreted with caution; evidence of coagulopathy is challenging to distinguish. The main points of interest are the peripheral smear, low platelet counts, affected coagulation function tests, high D-dimer levels, low fibrinogen levels and increased partial thromboplastin time, which, alongside prothrombin, may at times only be slightly altered or even appear within normal range $(4,140)$. Finally, limb venous duplex scans, echocardiograms and pulmonary ventilation perfusion scans are also useful modalities in the investigation of systemic coagulopathy (60).

NBTE is usually silent until severe complications occur, such as embolization; the incidence of cerebral ischemia is significantly higher than in infective endocarditis (61). The valvular lesions can lead to new-onset cardiac murmurs, arrhythmias and heart failure, with dyspnea, orthopnea and peripheral edemas (61). NBTE-induced stroke usually presents with focal or multifocal symptoms (23) and aphasia is considered to be the commonest neurological feature (59). The widespread infarctions may cause confusion and lethargy, and the encephalopathy may also fluctuate $(23,141)$.

The diagnosis of NBTE requires, first and foremost, the exclusion of infection (61). Transthoracic or transesophageal echocardiography can serve as the initial imaging processes for the assessment of the valvular lesions, which are usually rounded, sessile, heterogeneous in shape, and $>3 \mathrm{~mm}$ in size, and are located mainly on the mitral and aortic valves $(60,61,142)$. An MRI typically shows numerous infarctions of varying size in several territories $(61,143)$ and a CT scan/MRI is also useful in detecting neurovascular embolization (61). Angiography can also detect the occlusions with sensitivity, which are commonly located in the middle cerebral artery (59), and diffusion-weighted MRI can also assist in characterizing the stroke patterns (61). Ultimately, the most definite diagnosis can only occur postmortem (61).

Concerning infections, septic emboli may result in focal cerebral signs, seizures or encephalopathy and distinct manifestations depending on the microorganism $(56,68)$. In order to diagnose fungal infections in particular, a high index of clinical suspicion is crucial, since the isolation of the microorganism is difficult $(56,144)$. The diagnosis may require cultures and histopathology of tissue samples, fungal antigen assays or molecular tests for fungal DNA (144).

Leukostasis as a result of hyperleukocytosis usually affects the lungs and the CNS (145). In the CNS, it is dynamic and reversible, fluctuating according to the leukocyte count (50). The CNS symptoms range from nausea, tinnitus, visual impairment, ataxia, psychiatric manifestations such as agitation or delirium, to mental status disorders, with somnolence, stupor and even coma. The respiratory involvement varies and can lead to acute respiratory failure. Often, fever, leg or intestinal pain and priapism can occur (145). There is a lack of objective and definitive diagnostic criteria for leukostasis. The absolute number of WBC alone is not a criterion; it must be interpreted in the context of the underlying malignancy. Chest $\mathrm{X}$-rays or CT are not of much help; they correlate poorly to the clinical status. Cranial CT should be performed when CNS symptoms arise to exclude hemorrhage and evaluated alongside echocardiography, as this condition also heavily affects the right ventricle function (145).

\section{Detection of a cancer-associated stroke}

A concise presentation of the elements that hint towards the presence of a cancer-associated stroke is shown in Fig. 2. D-Dimers have gained significant ground in publications over the detection of a cancer-related stroke. In a 2014 study, patients with active cancer and ischemic stroke tended to demonstrate higher CRP and D-dimers, more frequent cryptogenic strokes and patterns of multiple lesions, and among those patients, higher CRP and D-dimer levels were associated with a cryptogenic mechanism and multiple lesion patterns (17). In agreement with that study, Xie et al analyzed data from patients with lung cancer and came to the conclusion that high D-Dimers, alongside CA125 and CA199, were independent risk factors for lung-cancer-associated stroke (146). In a different study, lung cancer was also associated with high CRP levels (7). High CRP and high fibrinogen have also been found in patients with occult malignancy that presented with a stroke. More specifically, Cocho et al (2015) reported that levels of CRP $>20 \mathrm{mg} / \mathrm{l}$ had a sensitivity of $75 \%$ and a 
specificity of $96 \%$, whereas fibrinogen levels $>600 \mathrm{mg} / \mathrm{dl}$ had a sensitivity of $67 \%$ and a specificity of $91 \%$ for occult malignancy presenting with a stroke. Therefore, it was suggested that screening be carried out in cancer patients exhibiting elevated levels of these two markers, particularly when the etiology of the stroke is unknown (28).

Karlińska et al (2015) also noted that patients with stroke with an active cancer tended to present lower hematocrit levels, higher serum CRP levels, and a higher erythrocyte sedimentation rate when compared to cancer-free stroke patients (27). Several other studies have also reported elevated D-Dimers levels in cancer patients $(7,19,146,147)$. Taking into consideration the significance D-Dimers seem to have in the diagnosis of cancer-related stroke, Guo et al (2014) used D-Dimers of $\geq 0.55 \mathrm{mg} / 1$, and multiple territory infarctions, as criteria for the development of a clinically meaningful test for cancer-associated stroke. The specificity and positive predictive value (PPV) for cancer-related stroke were 99.7 and $92.9 \%$, respectively. When the cut-off D-Dimers value was set at $\geq 5.5 \mathrm{mg} / 1$, the test had a high specificity and PPV regardless of the imaging results (148).

Nonetheless, the question of when a stroke patient should be screened for cancer remains. Selvik et al (2015) claimed that routine investigation for cancer was not justified in acute ischemic stroke (7). However, a number of studies have corroborate that systemic cancer workup should be considered in patients in whom stroke origin is unclear, who have an early vascular recurrence, predisposing factors for cancer, such as smoking, high D-Dimers, fibrinogen and CRP levels $(7,28,146)$. Xie et al suggested that patients with cryptogenic stroke and high plasma levels of CA125 and CA199 should also be investigated to rule out lung cancer (146). Regardless, stroke as the first manifestation of an underlying malignancy is mostly secondary to specific cancer-related causes (5). In a recent publication, Selvik et al (2018) developed a predictive score for clinical use, in order to uncover an underlying malignancy. The score consists of three elements, and when a patient fulfills one, it counts as one point. Specifically, D-dimers $\geq 3 \mathrm{mg} / \mathrm{l}, \mathrm{Hb} \leq 12.0 \mathrm{~g} / \mathrm{dl}$ and an active smoking or history of smoking give one point each. When a patient fulfills all 3 score points, the probability of active cancer is 53\%, and the overall test gave an area under the curve (AUC) of $73 \%$ for patients younger than 75 years of age (149).

As regards neuroimaging findings, cancer patients with stroke mostly have multiple lesions in multiple vascular territories $(1,61,150)$, a finding that has been used in developing clinical tests, as previously mentioned. Summarizing, neuroimaging studies, measurement of coagulation function, and echocardiography are the most useful modalities to identify the stroke $(23,31)$. In general, cancer-associated ischemic stroke is widely associated with lesions in multiple vascular territories $(1,61,150)$, elevated D-dimers and fibrin degradation products $(7,17,19,27,146,147,149,150)$, cancer markers (146) and proinflammatory components such as low hematocrit and high CRP levels $(7,27,28,149)$. It is up to the experience of the doctor to assess the patient using the aforementioned tools, when a high level of clinical suspicion is present; consequently, vigilance is essential. Several methods are currently being investigated to help the clinician to diagnose such a case, and they may be applied to every-day clinical practice in the near future.

\section{Management}

The clinical management and care of cancer patients with stroke differs from that of patients with stroke alone (3). The stroke extent depends on the activity and the severity of the cancer (17), and cancer patients have an inferior neurological condition at discharge and a tendency towards a longer stay in the stroke unit (15). The survival rate of patients hospitalized with cerebral infarction is worse in cancer patients than in those without cancer, and the outcome is poorer, and associated with both the severity of neurological disability and the tumor stage $(1,10,25,73)$. CVD in cancer patients is often aggressive, with a tendency to provoke recurrent events and rapid neurological devastation (62). Thus, adequate therapy in cancer patients with stroke may ameliorate the symptoms or prevent further episodes $(56,151)$. The management of specific occurrences in cancer patients is presented in Table II.

Acute stroke in patients with cancer can be treated with recombinant tissue plasminogen activator (rTPA), and the active cancer should not be seen as an absolute contraindication to rTPA use $(27,151,152)$. Clinical experience and small-scale trials, such as the one published by Cappellari et al (2013) have revealed that intravenous thrombolysis is not associated with a higher risk of hemorrhage in cancer patients, but rather improves the neurological state of such patients (151). The same findings were reported in an analysis by Sobolewski et al (2015) on intravenous thrombolysis, showing no impact of neoplastic disease on unfavorable outcomes (153).

Murthy et al (2013) reported that of the 32,576 stroke cases treated with thrombolysis, the cancer-associated stroke cases had significantly higher comorbidity indices, although there was no difference in the rates of home discharge and in-hospital mortality, after adjusting for confounders. Additionally, subgroup analysis revealed that compared with liquid cancers, patients with solid tumors had lower home discharge rates and higher in-hospital mortality. Metastatic cancers had the poorest outcomes, but the intracerebral hemorrhage rates were similar, implying that even in patients with metastatic cancer, thrombolysis remains the main therapeutic option (77). Thrombolysis is also considered safe for patients with primary brain tumors. Analysis did show that malignant brain tumors were tied to higher in-hospital mortality rate, lower home discharge and an overall increased risk of ICH, particularly when they had an intraparenchymal location, but thrombolysis did not present an additional risk for of ICH (154).

As for brain hemorrhage, the hemorrhage may require evacuation alongside with the additional antineoplastic treatment (56). The management of acute ICH in patients with cancer should conform to established guidelines for IPH, SAH, SDH, and EDH and in ITH, steroids could also be used to decrease mass effect from vasogenic edema, while the underlying tumor should further be considered for resection if surgically feasible (42).

For CVT, in some cases, observation alone may be adequate. Treatment with anticoagulation agents has been shown to be safe and may be beneficial in reducing mortality and long-term morbidity (33). As regards safety, anticoagulation agents could be administered even in the presence of a hematoma $(33,155)$. Mechanical clot thrombectomy has also been proven safe and effective (156); fibrinolytic or 
Table II. Management of specific occurrences in patients with cancer-associated stroke.

Occurrence

Indicated treatment

Ischemic stroke ${ }^{\mathrm{a}}$

Brain hemorrhage

Vasogenic edema

Cerebral vein thrombosis

Acute venous thromboembolism

Tumor-related venous occlusion

Leptomeningeal metastases

Intravascular lymphomatosis

NBTE

DIC

\author{
rTPA/IV thrombolysis \\ Evacuation, antineoplastic treatment \\ Corticosteroids, tumor resection \\ Observation, anticoagulation, mechanical clot thrombectomy, fibrinolytic or \\ endovascular therapy \\ LMWH \\ Brain radiation, chemotherapy, tumor resection \\ Irradiation, surgical extirpation, chemotherapy \\ Chemotherapy \\ Heparin, valvular repair or replacement \\ Categorization $^{\mathrm{b}}$, platelet transfusion, fresh-frozen plasma, cryoprecipitate or \\ fibrinogen concentrate administration ${ }^{\mathrm{c}}$, heparin or $\mathrm{LMWH}^{\mathrm{d}}$
}

rTPA, recombinant tissue plasminogen activator; IV, intravenous; LMWH, low-molecular-weight heparins; NBTE, non-bacterial thrombotic endocarditis; DIC, disseminated intravascular coagulation. ${ }^{a}$ The treatment can be applied even in patients with brain metastases or primary brain tumors; 'into procoagulant, hyperfibrinolytic or subclinical; ${ }^{c}$ depending on the subtype and clinical needs; ${ }^{\mathrm{d}}$ not for hyperfibrinolytic DIC.

endovascular therapy may even prove life-saving in critically ill patients (33). Low-molecular-weight heparins (LMWHs) constitute the current treatment of choice for cancer-associated acute venous thromboembolism $(157,158)$, although the evidence to support the superiority of heparin or LMWHs is still insufficient (33). The addition of aspirin or steroids is not recommended (33).

The treatment of tumor-related venous occlusion is typically brain radiation or chemotherapy, depending on the tumor type. Venous flow can be restored if therapy effectively treats the underlying tumor, and tumor resection, chemotherapy or radiation may be indicated for some large skull or dural tumors obstructing the sinus $(4,159)$.

Concerning leptomeningeal metastases, preferred treatments for select patients encompass irradiation, alongside surgical extirpation and chemotherapy. The radiotherapy is administered to the involved disease sites, while chemotherapy can be intra-CSF and systematic (29).

The prognosis of intravascular lymphomatosis (IVL) is poor $(37,38)$; CNS IVL in particular has a very poor survival rate when compared to non-CNS or skin IVL and the highest postmortem diagnosis rate (38); however, IVL is sensitive to systematic chemotherapy (38).

The current therapy for NBTE focuses on treating the underlying disease while managing the risk for systemic embolization $(23,61)$. Anticoagulation is crucial for preventing recurrent embolization and the American College of Chest Physician Guidelines recommend long-term anticoagulation, regardless of the evidence of emboli (61). Unfractionated heparin decreases the occurrence of thromboembolic events, particularly in patients with malignancy, and consequently, the rates of ischemic stroke, something that cannot be said about warfarin, which is not recommended $(23,61)$. Valvular repair or replacement has an indication in patients with severe dysfunction, large vegetations, recurrent embolism and no response to anticoagulation treatment, which should be explored prior to reaching a surgical decision. In any case, the clinician should weigh the benefits and risks, as the surgical mortality rate for these patients is high (61).

The management of DIC is a complex matter and should be individualized according to the clinical setting $(55,56)$. Recent guidance for cancer patients suggests, as a first step, the categorization of DIC into procoagulant, hyperfibrinolytic and subclinical. In general, appropriate management of the underlying malignancy remains key in its treatment. Specifically for DIC, platelet transfusions are recommended for a high risk of bleeding or active bleeding, which may also be further treated with the administration of fresh-frozen plasma, cryoprecipitate or fibrinogen concentrate. For the inhibition of the excess thrombin effects, heparin or LMWHs can be used as prophylactic therapy when no contraindications, such as low platelet count or active bleeding, are present. Subclinical types may also benefit from this prophylaxis, but it is not recommended for hyperfibrinolytic DIC (55).

The new oral anticoagulants (NOAGs) are nowadays considered an attractive option for coagulation prophylaxis and treatment; thus, discussing the efficiency of NOAGs in a cancer setting is appropriate. In a recent meta-analysis for the use of NOAGs in treating acute venous thromboembolism, NOAGs have been shown to have the same efficacy as Vitamin $\mathrm{K}$ antagonists in cancer patients and share many of the advantages of LMWHs, such as the short half-life (157). Accordingly, the safety of these drugs may also be considered in cancer patients with stroke. Furthermore, anticoagulants (enoxaparin) did not seem to aggravate the risk of $\mathrm{ICH}$ in patients with metastatic brain cancer, and did not pose an additional risk to patients with cancers that inherently present a higher risk for ICH, such as melanoma (21).

In conclusion, the clinician should carefully evaluate the risks and benefits of prescribing an oral anticoagulant to a patient with active malignancy. Further studies on their safety and efficacy on cancer patients are still required (160). Since they have not been compared directly to LMWHs, van Es and Büller (2015) suggested refraining from 
administering oral anticoagulants to cancer patients, though it may indeed be a good solution for patients not willing to endure constant injections (161). However, it must be said, that atrial fibrillation should still be considered over the cancer-induced hypercoagulability in patients with embolic appearing strokes, and, if discovered, anticoagulation should be initiated (26).

Finally, for carotid stenosis following RT, although there is a lack of large-scale studies, available literature to date estimates that revascularization surgery does not present a larger peri-operative risk than in regular angioplasty procedures $(56,162)$.

\section{Prevention}

Stroke is a serious condition that majorly impacts the prognosis in every patient, and particularly that of cancer patients. Therefore, measures to prevent it should be seriously considered. Furthermore, protective measures can also be taken in several conditions that are involved in a cancer-associated stroke. Firstly, cancer patients still present the same predisposing factors (high blood pressure, hyperlipidemia, diabetes mellitus, atrial fibrillation, carotid disease, smoking) with the rest of the population, so regulating those first and foremost is crucial (26). Secondly, cancer is generally accompanied by various complications that derive from the hypercoagulable state it creates. Thus, clinicians may need to administer anticoagulant treatment and the NOAGs are, as previously mentioned, an attractive option; per os consumption facilitates patient compliance and they offer a wide therapeutic window, requiring no laboratory monitoring $(160,161)$. However, some concern is raised due to their possible interaction with several chemotherapeutic agents and drugs used for supportive care through the CYP3A4 enzyme and/or P-glycoprotein transporter, and their possibly-impaired absorption in vomiting patients and patients with chemotherapy-induced intestinal mucosal defects $(157,160,161)$. Further caution is advised in cases of renal insufficiency that can occur in cancer patients, as serious and fatal bleeding complications can emerge in that setting (160). Another considerable drawback remains the lack of efficient measures to promptly reverse their anticoagulant activity in cases of overdose, bleeding, or other urgent indication for reversal (160).

Young adult pediatric cancer survivors have an increased stroke risk that is associated with cervical RT in a dose-dependent manner (103), implying that the lower the RT dose is, the lower the stroke risk becomes. Limiting neck radiation, wherever possible, in lymphoma patients, should consequently be considered (94). Routine duplex ultrasound screening is indicated for patients who had received RT to the head and neck (95). Fludeoxyglucose $\left({ }^{18} \mathrm{~F}\right)(18-\mathrm{FDG})$ positron emission tomography (PET)/CT successfully identified patients at risk for future vascular events in an otherwise asymptomatic cohort with neoplastic disease (163), so it could also be used to pinpoint the patients more prone towards stroke.

Concerning chemotherapy, agents that have anti-estrogenic effects, such as most endocrine therapies for breast cancer, may also increase the risk of CVD. Long-term adjuvant anastrozole treatment may result in significantly fewer thromboembolic cases and CVE when compared to tamoxifen (74). For chemotherapy-induced thrombocytopenia, which may predispose to hemorrhage, a delay in administration or a dose reduction of the chemotherapeutic drugs is usually the method of choice (54). With the exception of myeloablative therapy in hematologic malignancies, chemotherapy for non-hematologic ones rarely requires platelet transfusion support (54). An additional interesting finding to be mentioned is that paclitaxel presented a platelet-sparing effect in ovarian cancer patients that were treated with platinum regimens. In these patients, dose-dense carboplatin regimens were given as to overcome platinum resistance, but it led to high-grade thrombocytopenia (54).

\section{Conclusions}

Vigilance for stroke and CVD is always required in patients with cancer. CVD in cancer patients is often aggressive, with a tendency to provoke recurrent events and rapid neurological devastation; therefore, a prompt and accurate diagnosis is crucial. The vascular events in cancer patients can derive from a plethora of mechanisms that differ from non-cancer ones and include, among others, direct tumor effects, hypercoagulability, infections and the effects of the cancer-treatment, chemo- and radiotherapy.

Cancers with the highest incidences of stroke are lung, pancreatic, colorectal, breast and prostate. Though not as frequent but dangerous when it comes to strokes, are renal-cell carcinomas and melanomas, especially concerning hemorrhages.

The diagnosis of stroke and stroke-related situations entails several laboratory and imaging tests, following the clinician's suspicion based on the clinical presentation and patient history. Thus, when it comes to detecting a cancer-associated stroke, it is crucial that suspicion first arises. Cancer-associated ischemic strokes tend to be more multifocal and have consistently been tied to high D-Dimer plasma levels, and various other markers, such as CRP and fibrin degradation products. They are also associated with multifocal lesions in neuroimaging. As new data become available, more clinical tests and criteria for detecting these particular strokes are being developed.

The management of acute ischemic stroke has not been shown to differ largely in cancer and non-cancer patients; cancer should not be considered an absolute contraindication for intravenous thrombolysis. When it comes to prevention, the clinician should weigh the benefits and risks of an anticoagulation treatment plan and proceed with caution in administering it. So far, LMWHs remain the first choice.

In conclusion, cancer-associated stroke should be seriously evaluated. Clinicians should be aware of the risk, and upon development, able to face it efficiently, particularly when it complicates or occurs in the course of a malignancy, as these patients have been shown to have the worst clinical outcomes.

\section{Acknowledgements}

Not applicable.

\section{Funding}

MA was supported by the National Institute of Health (NIH) R01 ES10563, R01 ES07331 and R01 ES020852. This study was 
also supported by the Special Research Account of University of Crete (ELKE nos. 4602, 4920 and 3963) and ToxPlus S.A.

\section{Availability of data and materials}

Not applicable.

\section{Authors' contributions}

ED, AMA, SM, VS, DAS, PDM and AT were involved in the conception of the study. ED, AMA, SM, VS, KT, GT, ML, APK, AGB, MA, IL, DPB, DAS, PDM and AT were involved in the acquisition of the data and the study design, and were involved in the writing of the article and critically revised the manuscript.

\section{Ethics approval and consent to participate}

Not applicable.

\section{Patient consent for publication}

Not applicable.

\section{Competing interests}

DAS is the Editor-in-Chief for the journal, but had no personal involvement in the reviewing process, or any influence in terms of adjudicating on the final decision, for this article.

\section{References}

1. Kneihsl M, Enzinger C, Wünsch G, Khalil M, Culea V, Urbanic-Purkart T, Payer F, Niederkorn K, Fazekas F and Gattringer T: Poor short-term outcome in patients with ischaemic stroke and active cancer. J Neurol 263: 150-156, 2016.

2. Newton HB: Neurologic complications of systemic cancer. Am Fam Physician 59: 878-886, 1999.

3. Arboix A: Cerebrovascular disease in the cancer patient. Rev Neurol 31: 1250-1252, 2000 (In Spanish).

4. Grisold W, Oberndorfer S and Struhal W: Stroke and cancer: A review. Acta Neurol Scand 119: 1-16, 2009.

5. Taccone FS, Jeangette SM and Blecic SA: First-ever stroke as initial presentation of systemic cancer. J Stroke Cerebrovasc Dis 17: 169-174, 2008.

6. Selvik HA, Thomassen L, Logallo N and Næss H: Prior cancer in patients with ischemic stroke: The Bergen NORSTROKE study. J Stroke Cerebrovasc Dis 23: 919-925, 2014.

7. Selvik HA, Thomassen L, Bjerkreim AT and Næss $\mathrm{H}$ : Cancer-Associated Stroke: The Bergen NORSTROKE Study. Cerebrovasc Dis Extra 5: 107-113, 2015.

8. Sanossian N, Djabiras C, Mack WJ and Ovbiagele B: Trends in cancer diagnoses among inpatients hospitalized with stroke. J Stroke Cerebrovase Dis 22: 1146-1150, 2013.

9. Cestari DM, Weine DM, Panageas KS, Segal AZ and DeAngelis LM: Stroke in patients with cancer: Incidence and etiology. Neurology 62: 2025-2030, 2004.

10. Zhang YY, Chan DK, Cordato D, Shen Q and Sheng AZ: Stroke risk factor, pattern and outcome in patients with cancer. Acta Neurol Scand 114: 378-383, 2006

11. Graus F, Rogers LR and Posner JB: Cerebrovascular complications in patients with cancer. Medicine (Baltimore) 64: 16-35, 1985.

12. De Bruin ML, Dorresteijn LD, van't Veer MB, Krol AD, van der Pal HJ, Kappelle AC, Boogerd W, Aleman BM and van Leeuwen FE: Increased risk of stroke and transient ischemic attack in 5-year survivors of Hodgkin lymphoma. J Natl Cancer Inst 101: 928-937, 2009

13. Lindvig K, Møller H, Mosbech J and Jensen OM: The pattern of cancer in a large cohort of stroke patients. Int J Epidemiol 19: 498-504, 1990.
14. Bowers DC, McNeil DE, Liu Y, Yasui Y, Stovall M, Gurney JG, Hudson MM, Donaldson SS, Packer RJ, Mitby PA, et al: Stroke as a late treatment effect of Hodgkin's Disease: A report from the Childhood Cancer Survivor Study. J Clin Oncol 23: 6508-6515, 2005.

15. Stefan O, Vera N, Otto B, Heinz L and Wolfgang G: Stroke in cancer patients: A risk factor analysis. J Neurooncol 94: 221-226, 2009.

16. Zöller B, Ji J, Sundquist J and Sundquist K: Risk of haemorrhagic and ischaemic stroke in patients with cancer: A nationwide follow-up study from Sweden. Eur J Cancer 48: 1875-1883, 2012.

17. Lee EJ, Nah HW, Kwon JY, Kang DW, Kwon SU and Kim JS: Ischemic stroke in patients with cancer: Is it different from usual strokes? Int J Stroke 9: 406-412, 2014

18. Sheng B, Fong MK, Chu YP, Cheong AP, Teng SK, Chu JP, Yip WM and Chow DH: Stroke and cancer: Misfortunes never come singularly. Int J Stroke 8: E30, 2013.

19. Carrilho Romeiro A, Valadas A and Marques J: Acute ischemic stroke on cancer patients, a distinct etiology? A case-control study. Acta Med Port 28: 613-618, 2015.

20. Katz JM and Segal AZ: Incidence and etiology of cerebrovascular disease in patients with malignancy. Curr Atheroscler Rep 7: 280-288, 2005.

21. Donato J, Campigotto F, Uhlmann EJ, Coletti E, Neuberg D, Weber GM and Zwicker JI: Intracranial hemorrhage in patients with brain metastases treated with therapeutic enoxaparin: A matched cohort study. Blood 126: 494-499, 2015.

22. Rogers LR: Cerebrovascular complications in cancer patients. Neurol Clin 9: 889-899, 1991

23. Rogers LR: Cerebrovascular complications in patients with cancer. Semin Neurol 24: 453-460, 2004.

24. Navi BB, Reiner AS, Kamel H, Iadecola C, Elkind MS, Panageas KS and DeAngelis LM: Association between incident cancer and subsequent stroke. Ann Neurol 77: 291-300, 2015.

25. Aarnio K, Joensuu H, Haapaniemi E, Melkas S, Kaste M, Tatlisumak T and Putaala J: Cancer in young adults with ischemic stroke. Stroke 46: 1601-1606, 2015.

26. Dearborn JL, Urrutia VC and Zeiler SR: Stroke and cancer: A complicated relationship. J Neurol Transl Neurosci 2: 1039, 2014.

27. Karlińska AG, Gromadzka G, Karliński MA and Członkowska A: The activity of malignancy may determine stroke pattern in cancer patients. J Stroke Cerebrovasc Dis 24: 778-783, 2015.

28. Cocho D, Gendre J, Boltes A, Espinosa J, Ricciardi AC, Pons J, Jimenez M and Otermin P: Predictors of occult cancer in acute ischemic stroke patients. J Stroke Cerebrovasc Dis 24: 1324-1328, 2015.

29. Chamberlain MC: Leptomeningeal metastasis. Semin Neurol 30: 236-244, 2010

30. Herman C, Kupsky WJ, Rogers L, Duman R and Moore P: Leptomeningeal dissemination of malignant glioma simulating cerebral vasculitis. Case report with angiographic and pathological studies. Stroke 26: 2366-2370, 1995.

31. Raizer JJ and DeAngelis LM: Cerebral sinus thrombosis diagnosed by MRI and MR venography in cancer patients. Neurology 54: 1222-1226, 2000.

32. Klein P, Haley EC, Wooten GF and VandenBerg SR: Focal cerebral infarctions associated with perivascular tumor infiltrates in carcinomatous leptomeningeal metastases. Arch Neurol 46: 1149-1152, 1989

33. Ichord R: Cerebral sinovenous thrombosis. Front Pediatr 5: 163, 2017.

34. Ranta S, Tuckuviene R, Mäkipernaa A, Albertsen BK, Frisk T, Tedgård U, Jónsson ÓG, Pruunsild K, Gretenkort Andersson N, Winther Gunnes M, et al: Cerebral sinus venous thromboses in children with acute lymphoblastic leukaemia - a multicentre study from the Nordic Society of Paediatric Haematology and Oncology. Br J Haematol 168: 547-552, 2015.

35. Rogers LR: Cerebrovascular complications in patients with cancer. Semin Neurol 30: 311-319, 2010.

36. Santoro N, Giordano P, Del Vecchio GC, Guido G, Rizzari C, Varotto S, Masera G and De Mattia D: Ischemic stroke in children treated for acute lymphoblastic leukemia: A retrospective study. J Pediatr Hematol Oncol 27: 153-157, 2005.

37. Anghel G, Petrinato G, Severino A, Remotti D, Insabato L, De Renzo A, Rotoli B and Majolino I: Intravascular B-cell lymphoma: Report of two cases with different clinical presentation but rapid central nervous system involvement. Leuk Lymphoma 44: 1353-1359, 2003.

38. Fonkem E, Lok E, Robison D, Gautam S and Wong ET: The natural history of intravascular lymphomatosis. Cancer Med 3: 1010-1024, 2014 
39. Lefkovitz NW, Roessmann U and Kori SH: Major cerebral infarction from tumor embolus. Stroke 17: 555-557, 1986.

40. Zheng Z, Guo G, Xu L, Lei L, Wei X and Pan Y: Left atrial myxoma with versus without cerebral embolism: Length of symptoms, morphologic characteristics, and outcomes. Tex Heart Inst J 41: 592-595, 2014.

41. Murata J, Sawamura Y, Takahashi A, Abe H and Saitoh H: Intracerebral hemorrhage caused by a neoplastic aneurysm from small-cell lung carcinoma: Case report. Neurosurgery 32: 124-126, 1993.

42. Velander AJ, DeAngelis LM and Navi BB: Intracranial hemorrhage in patients with cancer. Curr Atheroscler Rep 14: 373-381, 2012.

43. Navi BB, Reichman JS, Berlin D, Reiner AS, Panageas KS, Segal AZ and DeAngelis LM: Intracerebral and subarachnoid hemorrhage in patients with cancer. Neurology 74: 494-501, 2010

44. Lieu AS, Hwang SL, Howng SL and Chai CY: Brain tumors with hemorrhage. J Formos Med Assoc 98: 365-367, 1999.

45. Fukushima S, Terasaki M, Tajima Y and Shigemori M: Granulocytic sarcoma: An unusual complication of acute promyelocytic leukemia causing cerebellar hemorrhage. Case report. J Neurosurg 105: 912-915, 2006.

46. Reichman J, Singer S, Navi B, Reiner A, Panageas K, Gutin PH and Deangelis LM: Subdural hematoma in patients with cancer. Neurosurgery 71: 74-79, 2012.

47. McIver JI, Scheithauer BW, Rydberg CH and Atkinson JL: Metastatic hepatocellular carcinoma presenting as epidural hematoma: Case report. Neurosurgery 49: 447-449, 2001.

48. Würthner JU, Köhler G, Behringer D, Lindemann A, Mertelsmann $\mathrm{R}$ and Lübbert M: Leukostasis followed by hemorrhage complicating the initiation of chemotherapy in patients with acute myeloid leukemia and hyperleukocytosis: A clinicopathologic report of four cases. Cancer 85: 368-374, 1999

49. Shiber JR and Fines RE: Cerebral hemorrhage due to hyperleukocytosis. J Emerg Med 40: 674-677, 2011.

50. Nowacki P, Fryze C, Zdziarska B, Zyluk B, Grzelec H and Dudzik T: Central nervous system leukostasis in patients with leukemias and lymphomas. Folia Neuropathol 33: 59-65, 1995.

51. Collins RC, Al-Mondhiry H, Chernik NL and Posner JB: Neurologic manifestations of intravascular coagulation in patients with cancer. A clinicopathologic analysis of 12 cases. Neurology 25: 795-806, 1975.

52. Schwarzbach CJ, Schaefer A, Ebert A, Held V, Bolognese M, Kablau M, Hennerici MG and Fatar M: Stroke and cancer: The importance of cancer-associated hypercoagulation as a possible stroke etiology. Stroke 43: 3029-3034, 2012.

53. Lee AY: Cancer and thromboembolic disease: Pathogenic mechanisms. Cancer Treat Rev 28: 137-140, 2002.

54. Liebman HA: Thrombocytopenia in cancer patients. Thromb Res 133 (Suppl 2): S63-S69, 2014.

55. Thachil J, Falanga A, Levi M, Liebman $H$ and Di Nisio $M$ Scientific and Standardization Committee of the International Society on Thrombosis and Hemostasis: Management of cancerassociated disseminated intravascular coagulation: Guidance from the SSC of the ISTH. J Thromb Haemost 13: 671-675, 2015.

56. Rogers LR: Cerebrovascular complications in cancer patients. Neurol Clin 21: 167-192, 2003.

57. Nguyen T and DeAngelis LM: Stroke in cancer patients. Curr Neurol Neurosci Rep 6: 187-192, 2006

58. Yagi T, Takahashi K, Tanikawa M, Seki M, Abe T and Suzuki N Fatal intracranial hemorrhage after intravenous thrombolytic therapy for acute ischemic stroke associated with cancer-related nonbacterial thrombotic endocarditis. J Stroke Cerebrovasc Dis 23: e413-e416, 2014.

59. Rogers LR, Cho ES, Kempin S and Posner JB: Cerebral infarction from non-bacterial thrombotic endocarditis. Clinical and pathological study including the effects of anticoagulation. Am J Med 83: 746-756, 1987

60. Reisner SA, Brenner B, Haim N, Edoute Y and Markiewicz W: Echocardiography in nonbacterial thrombotic endocarditis: From autopsy to clinical entity. J Am Soc Echocardiogr 13: 876-881, 2000 .

61. Liu J and Frishman WH: Nonbacterial Thrombotic Endocarditis: Pathogenesis, Diagnosis, and Management. Cardiol Rev 24 244-247, 2016.

62. Royter V and Cohen SN: Recurrent embolic strokes and cardiac valvular disease in a patient with non-small cell adenocarcinoma of lung. J Neurol Sci 241: 99-101, 2006.

63. Lopez JA, Ross RS, Fishbein MC and Siegel RJ: Nonbacterial thrombotic endocarditis: A review. Am Heart J 113: 773-784, 1987.
64. Steiner I: Nonbacterial thrombotic endocarditis - a study of 171 case reports. Cesk Patol 29: 58-60, 1993.

65. Edoute Y, Haim N, Rinkevich D, Brenner B and Reisner SA: Cardiac valvular vegetations in cancer patients: A prospective echocardiographic study of 200 patients. Am J Med 102: 252-258, 1997

66. Correale JD, Monteverde DA, Bueri JA, Reich EG and Lucatelli N: Craniocerebral involvement in lymphoma. Arq Neuropsiquiatr 48: 306-314, 1990.

67. Noje C, Cohen K and Jordan LC: Hemorrhagic and ischemic stroke in children with cancer. Pediatr Neurol 49: 237-242, 2013.

68. Zembower TR: Epidemiology of infections in cancer patients. Cancer Treat Res 161: 43-89, 2014.

69. Bos MM, Smeets LS, Dumay I and de Jonge E: Bloodstream infections in patients with or without cancer in a large community hospital. Infection 41: 949-958, 2013.

70. Fugate JE, Lyons JL, Thakur KT, Smith BR, Hedley-Whyte ET and Mateen FJ: Infectious causes of stroke. Lancet Infect Dis 14: 869-880, 2014

71. Saynak M, Cosar-Alas R, Yurut-Caloglu V, Caloglu M, Kocak Z and Uzal C: Chemotherapy and cerebrovascular disease. J BUON 13: 31-36, 2008.

72. Abt NB, Bydon M, De la Garza-Ramos R, McGovern K, Olivi A, Huang $\mathrm{J}$ and Bydon A: Concurrent neoadjuvant chemotherapy is an independent risk factor of stroke, all-cause morbidity, and mortality in patients undergoing brain tumor resection. J Clin Neurosci 21: 1895-1900, 2014.

73. Wall JG, Weiss RB, Norton L, Perloff M, Rice MA, Korzun AH and Wood WC: Arterial thrombosis associated with adjuvant chemotherapy for breast carcinoma: A Cancer and Leukemia Group B Study. Am J Med 87: 501-504, 1989.

74. Esteva FJ and Hortobagyi GN: Comparative assessment of lipid effects of endocrine therapy for breast cancer: Implications for cardiovascular disease prevention in postmenopausal women. Breast 15: 301-312, 2006.

75. Robinson D, Garmo H, Lindahl B, Van Hemelrijck M, Adolfsson J, Bratt O, Holmberg L and Stattin P: Ischemic heart disease and stroke before and during endocrine treatment for prostate cancer in PCBaSe Sweden. Int J Cancer 130: 478-487, 2012.

76. Mosca L, Grady D, Barrett-Connor E, Collins P, Wenger N, Abramson BL, Paganini-Hill A, Geiger MJ, Dowsett SA, Amewou-Atisso M, et al: Effect of raloxifene on stroke and venous thromboembolism according to subgroups in postmenopausal women at increased risk of coronary heart disease. Stroke 40: 147-155, 2009.

77. Murthy SB, Karanth S, Shah S, Shastri A, Rao CP, Bershad EM and Suarez JI: Thrombolysis for acute ischemic stroke in patients with cancer: A population study. Stroke 44: 3573-3576, 2013

78. Ligibel JA, James O'Malley A, Fisher M, Daniel GW, Winer EP and Keating NL: Risk of myocardial infarction, stroke, and fracture in a cohort of community-based breast cancer patients. Breast Cancer Res Treat 131: 589-597, 2012.

79. Jagsi R, Griffith KA, Koelling T, Roberts R and Pierce LJ: Stroke rates and risk factors in patients treated with radiation therapy for early-stage breast cancer. J Clin Oncol 24: 2779-2785, 2006.

80. Rosell J, Nordenskjöld B, Bengtsson NO, Fornander T, Hatschek T, Lindman H, Malmström PO, Wallgren A, Stål O, et al: Time dependent effects of adjuvant tamoxifen therapy on cerebrovascular disease: Results from a randomised trial. Br J Cancer 104: 899-902, 2011.

81. Yang TL, Wu TC, Huang CC, Huang PH, Chung CM, Lin SJ, Chen JW, Chan WL, Chiang CH and Leu HB: Association of tamoxifen use and reduced cardiovascular events among asian females with breast cancer. Circ J 78: 135-140, 2014.

82. Azoulay L, Yin H, Benayoun S, Renoux C, Boivin JF and Suissa S: Androgen-deprivation therapy and the risk of stroke in patients with prostate cancer. Eur Urol 60: 1244-1250, 2011.

83. Jespersen CG, Nørgaard M and Borre M: Androgen-deprivation therapy in treatment of prostate cancer and risk of myocardial infarction and stroke: A nationwide Danish population-based cohort study. Eur Urol 65: 704-709, 2014.

84. Chung SD, Chen YK, Wu FJ and Lin HC: Hormone therapy for prostate cancer and the risk of stroke: A 5-year follow-up study. BJU Int 109: 1001-1005, 2012.

85. Meng F, Zhu S, Zhao J, Vados L, Wang L, Zhao Y, Zhao D and Niu Y: Stroke related to androgen deprivation therapy for prostate cancer: A meta-analysis and systematic review. BMC Cancer 16: 180, 2016.

86. Gerl A: Vascular toxicity associated with chemotherapy for testicular cancer. Anticancer Drugs 5: 607-614, 1994. 
87. Kuan AS, Teng CJ, Wu HH, Su VY, Chen YT, Chien SH, Yeh CM, Hu LY, Chen TJ, Tzeng CH, et al: Risk of ischemic stroke in patients with ovarian cancer: A nationwide populationbased study. BMC Med 12: 53, 2014.

88. Rollins N, Winick N, Bash R and Booth T: Acute methotrexate neurotoxicity: Findings on diffusion-weighted imaging and correlation with clinical outcome. AJNR Am J Neuroradiol 25 1688-1695, 2004.

89. Jourdan E, Dombret H, Glaisner S, Micléa JM, Castaigne S and Degos L: Unexpected high incidence of intracranial subdural haematoma during intensive chemotherapy for acute myeloid leukaemia with a monoblastic component. Br J Haematol 89: 527-530, 1995

90. Lee CW, Muo CH, Liang JA, Sung FC and Kao CH: Association of intensive morphine treatment and increased stroke incidence in prostate cancer patients: A population-based nested casecontrol study. Jpn J Clin Oncol 43: 776-781, 2013.

91. Stewart FA, Hoving S and Russell NS: Vascular damage as an underlying mechanism of cardiac and cerebral toxicity in irradiated cancer patients. Radiat Res 174: 865-869, 2010.

92. Tsai SJ, Huang YS, Tung CH, Lee CC, Lee MS, Chiou WY, Lin HY, Hsu FC, Tsai CH, Su YC, et al: Increased risk of ischemic stroke in cervical cancer patients: A nationwide population-based study. Radiat Oncol 8: 41, 2013.

93. Santoro F, Tarantino N, Pellegrino PL, Caivano M, Lopizzo A, Di Biase M and Brunetti ND: Cardiovascular sequelae of radiation therapy. Clin Res Cardiol 103: 955-967, 2014.

94. Scott AS, Parr LA and Johnstone PA: Risk of cerebrovascular events after neck and supraclavicular radiotherapy: A systematic review. Radiother Oncol 90: 163-165, 2009.

95. Cheng SW, Wu LL, Ting AC, Lau H, Lam LK and Wei WI: Irradiation-induced extracranial carotid stenosis in patients with head and neck malignancies. Am J Surg 178: 323-328, 1999.

96. Elerding SC, Fernandez RN, Grotta JC, Lindberg RD, Causay LC and McMurtrey MJ: Carotid artery disease following external cervical irradiation. Ann Surg 194: 609-615, 1981.

97. Dorresteijn LD, Kappelle AC, Boogerd W, Klokman WJ, Balm AJ, Keus RB, van Leeuwen FE and Bartelink H: Increased risk of ischemic stroke after radiotherapy on the neck in patients younger than 60 years. J Clin Oncol 20: 282-288, 2002.

98. Smith GL, Smith BD, Buchholz TA, Giordano SH, Garden AS, Woodward WA, Krumholz HM, Weber RS, Ang KK and Rosenthal DI: Cerebrovascular disease risk in older head and neck cancer patients after radiotherapy. J Clin Oncol 26: 5119-5125, 2008.

99. Hung SK, Lee MS, Chiou WY, Lee CC, Chen YC, Lai CL, Chien NC, Hsu WL, Liu DW, Su YC, et al: High incidence of ischemic stroke occurrence in irradiated lung cancer patients: A population-based surgical cohort study. PLoS One 9: e94377, 2014.

100. Woodward WA, Giordano SH, Duan Z, Hortobagyi GN and Buchholz TA: Supraclavicular radiation for breast cancer does not increase the 10-year risk of stroke. Cancer 106: 2556-2562, 2006

101. Morris B, Partap S, Yeom K, Gibbs IC, Fisher PG and King AA Cerebrovascular disease in childhood cancer survivors: A Children's Oncology Group Report. Neurology 73: 1906-1913, 2009.

102. Mueller S, Sear K, Hills NK, Chettout N, Afghani S, Gastelum E, Haas-Kogan D and Fullerton HJ: Risk of first and recurrent stroke in childhood cancer survivors treated with cranial and cervical radiation therapy. Int J Radiat Oncol Biol Phys 86: 643-648, 2013.

103. Mueller S, Fullerton HJ, Stratton K, Leisenring W, Weathers RE, Stovall M, Armstrong GT, Goldsby RE, Packer RJ, Sklar CA, et al: Radiation, atherosclerotic risk factors, and stroke risk in survivors of pediatric cancer: A report from the Childhood Cancer Survivor Study. Int J Radiat Oncol Biol Phys 86: 649-655, 2013.

104.Ruggiu M, Cuccuini W, Mokhtari K, Meignin V, Peffault de Latour R, Robin M, Fontbrune FS, Xhaard A, Socié G, et al: Case report: Central nervous system involvement of human graft versus host disease: Report of 7 cases and a review of literature. Medicine (Baltimore) 96: e8303, 2017.

105.Najima Y, Ohashi K, Miyazawa M, Nakano M, Kobayashi T, Yamashita $\mathrm{T}$, Akiyama $\mathrm{H}$ and Sakamaki $\mathrm{H}$ : Intracrania hemorrhage following allogeneic hematopoietic stem cell transplantation. Am J Hematol 84: 298-301, 2009.

106. Syed FI, Couriel DR, Frame D and Srinivasan A: Central nervous system complications of hematopoietic stem cell transplant. Hematol Oncol Clin North Am 30: 887-898, 2016.
107. Grauer O, Wolff D, Bertz H, Greinix H, Kühl JS, Lawitschka A, Lee SJ, Pavletic SZ, Holler E and Kleiter I: Neurological manifestations of chronic graft-versus-host disease after allogeneic haematopoietic stem cell transplantation: Report from the Consensus Conference on Clinical Practice in chronic graft-versus-host disease. Brain 133: 2852-2865, 2010.

108. Saad AG, Alyea EP III, Wen PY, Degirolami U and Kesari S: Graft-versus-host disease of the CNS after allogeneic bone marrow transplantation. J Clin Oncol 27: e147-e149, 2009.

109. Maffini E, Festuccia M, Brunello L, Boccadoro M, Giaccone L and Bruno B: Neurologic Complications after Allogeneic Hematopoietic Stem Cell Transplantation. Biol Blood Marrow Transplant 23: 388-397, 2017.

110. Goddeau RP and Alhazzani A: Headache in stroke: A review. Headache 53: 1019-1022, 2013.

111. Yew KS and Cheng EM: Diagnosis of acute stroke. Am Fam Physician 91: 528-536, 2015.

112. Leiva-Salinas $C$ and Wintermark M: Imaging of acute ischemic stroke. Neuroimaging Clin N Am 20: 455-468, 2010.

113. Liu YC, Lin TA, Ko PS, Wang HY, Chien SH, Liu CJ, Chiou TJ, Hsiao LT, Gau JP and Liu JH: Cerebrovascular disease after allogeneic hematopoietic stem cell transplantation: Incidence, manifestations, risk and clinical outcome. Blood 130: 4527-4527, 2017.

114. Zhang XH, Wang QM, Chen H, Chen YH, Han W, Wang FR, Wang JZ, Zhang YY, Mo XD, Chen Y, et al: Clinical characteristics and risk factors of Intracranial hemorrhage in patients following allogeneic hematopoietic stem cell transplantation. Ann Hematol 95: 1637-1643, 2016.

115. Bennett CL, Djulbegovic B, Norris LB and Armitage JO: Colony-stimulating factors for febrile neutropenia during cance therapy. N Engl J Med 368: 1131-1139, 2013.

116. McCune JS, Sullivan SD, Blough DK, Clarke L, McDermott C, Malin J and Ramsey S: Colony-stimulating factor use and impact on febrile neutropenia among patients with newly diagnosed breast, colorectal, or non-small cell lung cancer who were receiving chemotherapy. Pharmacotherapy 32: 7-19, 2012.

117. Rizzo JD, Brouwers M, Hurley P, Seidenfeld J, Somerfield MR and Temin S: American society of clinical oncology/american society of hematology clinical practice guideline update on the use of epoetin and darbepoetin in adult patients with cancer. J Oncol Pract 6: 317-320, 2010.

118. Kreys ED, Kim TY, Delgado A and Koeller JM: Impact of cancer supportive care pathways compliance on emergency department visits and hospitalizations. J Oncol Pract 10: 168-173, 2014

119. Poonawalla IB, Piller LB, Lairson DR, Chan W and Du XL: Impact of hematopoietic growth factors on blood transfusion needs, incidence of neutropenia, and overall survival among elderly advanced ovarian cancer patients treated with chemotherapy. Int J Gynecol Cancer 26: 95-103, 2016.

120. Du XL and Zhang Y: Risks of venous thromboembolism, stroke, heart disease, and myelodysplastic syndrome associated with hematopoietic growth factors in a large population-based cohort of patients with colorectal cancer. Clin Colorectal Cancer 14: e21-e31, 2015

121. Du XL, Zhang Y and Hardy D: Associations between hematopoietic growth factors and risks of venous thromboembolism, stroke, ischemic heart disease and myelodysplastic syndrome: Findings from a large population-based cohort of women with breast cancer. Cancer Causes Control 27: 695-707, 2016.

122. Puhalla S, Bhattacharya S and Davidson NE: Hematopoietic growth factors: Personalization of risks and benefits. Mol Oncol 6: 237-241, 2012

123. Calip GS, Malmgren JA, Lee WJ, Schwartz SM and Kaplan HG: Myelodysplastic syndrome and acute myeloid leukemia following adjuvant chemotherapy with and without granulocyte colony-stimulating factors for breast cancer. Breast Cancer Res Treat 154: 133-143, 2015.

124. Rosenstock AS, Niu J, Giordano SH, Zhao H, Wolff AC and Chavez-MacGregor M: Acute myeloid leukemia and myelodysplastic syndrome after adjuvant chemotherapy: A population-based study among older breast cancer patients. Cancer 124: 899-906, 2018.

125. Salamin O, Kuuranne T, Saugy $M$ and Leuenberger $N$ : Erythropoietin as a performance-enhancing drug: Its mechanistic basis, detection, and potential adverse effects. Mol Cell Endocrinol 464: 75-87, 2018

126. Bian J, Chen B, Hershman DL, Marks N, Norris L, Schulz R and Bennett CL: Effects of the US food and drug administration boxed warning of erythropoietin-stimulating agents on utilization and adverse outcome. J Clin Oncol 35: 1945-1951, 2017. 
127.Hollingsworth K, Romney MC, Crawford A and McAna J: The impact of the risk evaluation mitigation strategy for erythropoiesis-stimulating agents on their use and the incidence of stroke in medicare subjects with chemotherapy-induced anemia with lung and/or breast cancers. Popul Health Manag 19: 63-69, 2016.

128.Palmer SC, Saglimbene V, Mavridis D, Salanti G, Craig JC, Tonelli M, Wiebe N and Strippoli GF: Erythropoiesis-stimulating agents for anaemia in adults with chronic kidney disease: A network meta-analysis. Cochrane Database Syst Rev: CD010590, 2014.

129. Seliger SL, Zhang AD, Weir MR, Walker L, Hsu VD, Parsa A, Diamantidis CJ and Fink JC: Erythropoiesis-stimulating agents increase the risk of acute stroke in patients with chronic kidney disease. Kidney Int 80: 288-294, 2011.

130. Szczech L: Chronic kidney disease: Association between ESA use and stroke in patients with CKD. Nat Rev Nephrol 7: 365-366, 2011.

131.England TJ, Sprigg N, Alasheev AM, Belkin AA, Kumar A, Prasad K and Bath PM: Granulocyte-Colony Stimulating Factor (G-CSF) for stroke: An individual patient data meta-analysis. Sci Rep 6: 36567, 2016

132. Fan ZZ, Cai HB, Ge ZM, Wang LQ, Zhang XD, Li L and Zhai XB: The efficacy and safety of granulocyte colonyStimulating factor for patients with stroke. J Stroke Cerebrovasc Dis 24: 1701-1708, 2015.

133. Huang X, Liu Y, Bai S, Peng L, Zhang B and Lu H: Granulocyte colony stimulating factor therapy for stroke: A pairwise metaanalysis of randomized controlled trial. PLoS One 12: e0175774 2017.

134. Jacob L and Kostev K: Cancer is associated with intraoperative and postprocedural complications and disorders. J Cancer Res Clin Oncol 142: 777-781,2016.

135. Kamiya-Matsuoka C, Cachia D, Yust-Katz S, Rodriguez YA, Garciarena P, Rodarte EM and Tremont-Lukats IW; Anderson Cancer Center: Ischemic stroke in patients with gliomas at The University of Texas-M.D. J Neurooncol 125: 143-148, 2015.

136. Chen CW, Cheng TJ, Ho CH, Wang JJ, Weng SF, Hou YC, Cheng HC, Chio CC, Shan YS and Chang WT: Increased risk of brain cancer incidence in stroke patients: A clinical case series, population-based and longitudinal follow-up study. Oncotarget 8: 108989-108999, 2017.

137. Sealy-Jefferson S, Cote ML, Chlebowski RT, Rexrode KM and Simon MS: Post-stroke cancer risk among postmenopausal women: The Women's Health Initiative. Womens Health Issues 28 29-34, 2018.

138. Del Ben M, Caporale A, Feole K, Alessandri C and Angelico F: Intracranial hemorrage due to brain metastases in an Italian HCV patient with hepatocellular carcinoma. J Exp Clin Cancer Res 22: 641-644, 2003

139. Ponzoni M, Ferreri AJ, Campo E, Facchetti F, Mazzucchelli L, Yoshino T, Murase T, Pileri SA, Doglioni C, Zucca E, et al: Definition, diagnosis, and management of intravascular large B-cell lymphoma: Proposals and perspectives from an international consensus meeting. J Clin Oncol 25: 3168-3173, 2007.

140. Bal S, Menon B and Demchuk A: Relationship of endocarditis, disseminated intravascular coagulation, and embolic signals in cancer with stroke. Ann Neurol 71: 146, 2012.

141. Reagan TJ and Okazaki H: The thrombotic syndrome associated with carcinoma. A clinical and neuropathologic study. Arch Neurol 31: 390-395, 1974.

142.el-Shami K, Griffiths E and Streiff M: Nonbacterial thrombotic endocarditis in cancer patients: Pathogenesis, diagnosis, and treatment. Oncologist 12: 518-523, 2007.

143. Singhal AB, Topcuoglu MA and Buonanno FS: Acute ischemic stroke patterns in infective and nonbacterial thrombotic endocarditis: A diffusion-weighted magnetic resonance imaging study. Stroke 33: 1267-1273, 2002.

144. Angarone M: Fungal infections in cancer patients. Cancer Treat Res 161: 129-155, 2014

145. Schellongowski $\mathrm{P}$ and Staudinger T: Leukostasis and tumor lysis: Important complications of hyperleukocytosis. Internist (Berl) 54: 1051-1060,2013 (In German).

146. Xie X, Chen L, Zeng J, Qin C, Cheng D, Wei X and Liang Z: Clinical features and biological markers of lung cancer-associated stroke. J Int Med Res 44: 1483-1491, 2016.
147. Kono T, Ohtsuki T, Hosomi N, Takeda I, Aoki S, Sueda Y, Ishihara K, Nakamura T, Yamawaki T and Matsumoto M: Cancer-associated ischemic stroke is associated with elevated $\mathrm{D}$-dimer and fibrin degradation product levels in acute ischemic stroke with advanced cancer. Geriatr Gerontol Int 12: 468-474, 2012.

148. Guo YJ, Chang MH, Chen PL, Lee YS, Chang YC and Liao YC: Predictive value of plasma (D)-dimer levels for cancer-related stroke: A 3-year retrospective study. J Stroke Cerebrovasc Dis 23: e249-e254, 2014.

149. Selvik HA, Bjerkreim AT, Thomassen L, Waje-Andreassen U, Naess H and Kvistad CE: When to Screen Ischaemic Stroke Patients for Cancer. Cerebrovasc Dis 45: 42-47, 2018.

150. Kim SJ, Park JH, Lee MJ, Park YG, Ahn MJ and Bang OY: Clues to occult cancer in patients with ischemic stroke. PLoS One 7: e44959, 2012

151. Cappellari M, Carletti M, Micheletti N, Tomelleri G, Ajena D, Moretto $\mathrm{G}$ and Bovi P: Intravenous alteplase for acute ischemic stroke in patients with current malignant neoplasm. J Neurol Sci 325: 100-102, 2013.

152. Graber JJ, Nayak L and Deangelis LM: Use of recombinant tissue plasminogen activator in cancer patients with acute stroke. J Neurooncol 107: 571-573, 2012.

153. Sobolewski P, Brola W, Szczuchniak W, Fudala M and Sobota A: Safety of intravenous thrombolysis for acute ischaemic stroke including concomitant neoplastic disease sufferers - experience from Poland. Int J Clin Pract 69: 666-673, 2015.

154. Murthy SB, Moradiya Y, Shah S, Shastri A, Bershad EM and Suarez JI: In-hospital outcomes of thrombolysis for acute ischemic stroke in patients with primary brain tumors. J Clin Neurosci 22: 474-478, 2015

155. Fink JN and McAuley DL: Safety of anticoagulation for cerebral venous thrombosis associated with intracerebral hematoma. Neurology 57: 1138-1139, 2001.

156. Soleau SW, Schmidt R, Stevens S, Osborn A and MacDonald JD Extensive experience with dural sinus thrombosis. Neurosurgery 52 : 534-544, 2003

157. van der Hulle T, den Exter PL, Kooiman J, van der Hoeven JJ, Huisman MV and Klok FA: Meta-analysis of the efficacy and safety of new oral anticoagulants in patients with cancer-associated acute venous thromboembolism. J Thromb Haemost 12: 1116-1120, 2014

158. Raskob GE, van Es N, Verhamme P, Carrier M, Di Nisio M, Garcia D, Grosso MA, Kakkar AK, Kovacs MJ, Mercuri MF, et al; Hokusai VTE Cancer Investigators: Edoxaban for the Treatment of Cancer-Associated Venous Thromboembolism. N Engl J Med 378: 615-624, 2018.

159. Matsumoto K, Ohta $\mathrm{M}$ and Takeshita I: Reversible nonthrombotic occlusion of the superior sagittal sinus caused by metastatic malignant lymphoma - case report. Neurol Med Chir (Tokyo) 43: 349-351, 2003.

160. Short NJ and Connors JM: New oral anticoagulants and the cancer patient. Oncologist 19: 82-93, 2014.

161.van Es N and Büller HR: Using direct oral anticoagulants (DOACs) in cancer and other high-risk populations. Hematology Am Soc Hematol Educ Program 2015: 125-131, 2015.

162. Magne JL, Pirvu A, Sessa C, Cochet E, Blaise H and Ducos C: Carotid artery revascularisation following neck irradiation: Immediate and long-term results. Eur J Vasc Endovasc Surg 43: 4-7, 2012.

163. Rominger A, Saam T, Wolpers S, Cyran CC, Schmidt M, Foerster S, Nikolaou K, Reiser MF, Bartenstein P and Hacker M: ${ }^{18} \mathrm{~F}$-FDG PET/CT identifies patients at risk for future vascular events in an otherwise asymptomatic cohort with neoplastic disease. J Nucl Med 50: 1611-1620, 2009.

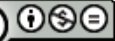

This work is licensed under a Creative Commons Attribution-NonCommercial-NoDerivatives 4.0 International (CC BY-NC-ND 4.0) License. 